\title{
Weak solutions of inverse mean curvature flow for hypersurfaces with boundary
}

\section{Journal Article}

Author(s):

Marquardt, Thomas

Publication date:

2017-07

Permanent link:

https://doi.org/10.3929/ethz-b-000270977

Rights / license:

In Copyright - Non-Commercial Use Permitted

Originally published in:

Journal für die reine und angewandte Mathematik 2017(728), https://doi.org/10.1515/crelle-2014-0116 


\title{
Weak solutions of inverse mean curvature flow for hypersurfaces with boundary
}

\author{
By Thomas Marquardt at Zurich
}

\begin{abstract}
We consider the evolution of hypersurfaces with boundary under inverse mean curvature flow. The boundary condition is of Neumann type, i.e. the evolving hypersurface moves along, but stays perpendicular to, a fixed supporting hypersurface. In this setup, we prove existence and uniqueness of weak solutions. Furthermore, we indicate the existence of a monotone quantity which is the analog of the Hawking mass for closed hypersurfaces.
\end{abstract}

\section{Introduction}

The evolution of closed hypersurfaces under inverse mean curvature flow (IMCF) was put forward by Geroch [5] and Jang and Wald [13] as an approach to the proof of the positive mass theorem. They observed the monotonicity of the Hawking mass under IMCF and showed that if IMCF remained smooth, this monotonicity could be used to prove the Riemannian Penrose inequality and thus the positive mass theorem. Unfortunately, IMCF does not remain smooth in general. However, the Positive Mass Theorem was proved by Schoen and Yau [26] using a different approach.

Later Gerhardt [4] showed that starting IMCF from a closed, smooth, star-shaped hypersurface with strictly positive mean curvature the surface evolves for all time and approaches a round sphere as time tends to infinity (see also Urbas [33]). For non-star-shaped initial hypersurfaces singularities may occur in finite time. In order to make sense of the flow in that situation Huisken and Ilmanen [11,12] defined weak solutions of IMCF which still keep the Hawking mass monotone. This enabled them to prove the Riemannian Penrose inequality. See also Bray [2] for a different approach.

Here we want to consider IMCF in the case where the hypersurfaces possess a boundary and move along, but stay perpendicular to, a fixed supporting hypersurface. For a short time this flow admits a classical solution [22]. Moreover, in the special case where the supporting hypersurface is a convex cone and the initial hypersurface is star-shaped and has strictly positive mean curvature we proved long-time existence and convergence to a piece of a round

This work was mainly supported by the Max Planck Institute for Gravitational Physics in Potsdam. The manuscript was prepared while the author was at ETH in Zurich where he received support from the Swiss National Science Foundation SNF 200021-140467. 
sphere [23]. The aim of this work is to prove the existence of weak solutions of IMCF for hypersurfaces with boundary. We use a level set approach along the ideas of Huisken and Ilmanen together with new barrier constructions and a priori estimates for a family of mixed Dirichlet-Neumann problems in non-smooth domains.

The article is organized as follows: In Section 2 we introduce the classical formulation of IMCF and describe the corresponding level set problem and a family of approximating problems. In Section 3 we derive a priori estimates for solutions of the approximating problems. This yields existence and uniqueness for solutions of these problems in weighted Hölder spaces. In Section 4 we define weak solutions of IMCF for hypersurfaces with boundary. Furthermore, we show that a sequence of solutions of the approximating problems converges to a weak solution. The main result is summarized in Theorem 4.8. We use Section 5 to discuss further properties of weak solutions and finish with Section 6 by proving the existence of a monotone quantity for classical solutions in Proposition 6.1. It is the analog of the Hawking mass for closed hypersurfaces. The justification of the monotonicity in the weak setting will be carried out in a forthcoming paper.

Acknowledgement. The author wants to thank Gerhard Huisken for acquainting him with inverse mean curvature flow and for all the support and valuable discussions during the time the author spent at the Max Planck Institute for Gravitational Physics in Potsdam.

\section{Level-set description and approximation}

Let us first consider the classical formulation of inverse mean curvature flow in the case where the hypersurfaces possess a boundary and move along, but stay perpendicular to a fixed supporting hypersurface.

Definition 2.1. Let $M^{n}$ be a compact, smooth, $n$-dimensional manifold with compact, smooth boundary $\partial M^{n}$. Let $\Sigma^{n}$ be a $C^{2, \alpha}$-hypersurface in $\mathbb{R}^{n+1}$ without boundary. Furthermore, let us assume that the initial hypersurface is described by an immersion $F_{0}: M^{n} \rightarrow \mathbb{R}^{n+1}$ such that $M_{0}^{n}:=F_{0}\left(M^{n}\right)$ has strictly positive mean curvature with respect to the unit normal vector field $v$ and satisfies

$$
F_{0}\left(\partial M^{n}\right)=F_{0}\left(M^{n}\right) \cap \Sigma^{n}, \quad\left\langle\nu, \mu \circ F_{0}\right\rangle=0 \quad \text { on } \partial M^{n}
$$

where $\mu$ is the unit normal vector fields of $\Sigma^{n}$. Given a one-parameter family of immersions $F: M^{n} \times[0, T) \rightarrow \mathbb{R}^{n+1}$, we say that $M_{t}^{n}:=F\left(M^{n}, t\right)$ moves under inverse mean curvature flow if $F$ satisfies

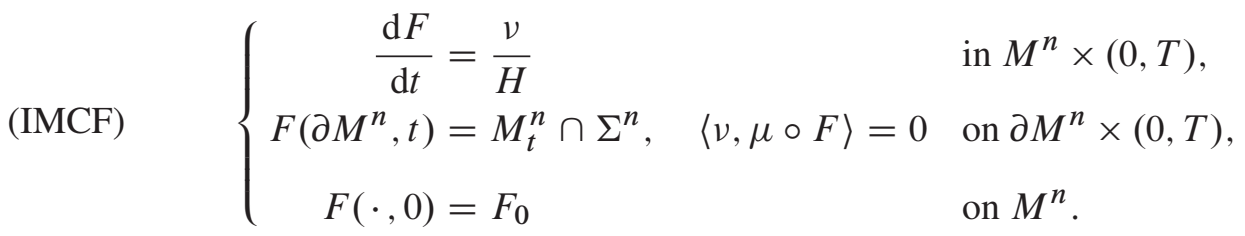

Here $v$ is the unit normal vector field on $M_{t}^{n}$ which is compatible with that of $M_{0}^{n}$ and $H$ is the scalar mean curvature ${ }^{1)}$ of $M_{t}^{n}$ in $\mathbb{R}^{n+1}$. Furthermore, $\mu$ is chosen to point away from $M_{t}^{n}$, i.e. for curves in $M_{t}^{n}$ ending at $p \in \partial M_{t}^{n}$ with tangent vector $v(p)$ we have $\langle v, \mu\rangle(p) \geq 0$.

1) Note that a classical solution of (IMCF) can only exists as long as $H$ remains strictly positive. 
In order to obtain the level set description we define the set of all points on one side of $\Sigma^{n}$ including $\Sigma^{n}$ itself:

$$
\begin{aligned}
\Omega:=\left\{x \in \mathbb{R}^{n+1} \mid \exists \gamma \in C^{1}\left([0,1], \mathbb{R}^{n+1}\right): \gamma(0)\right. & =\gamma([0,1]) \cap \Sigma^{n}, \\
\gamma(1) & \left.=x, \gamma^{\prime}(0)=-\mu\right\} \cup \Sigma^{n} .
\end{aligned}
$$

Furthermore, for a set $A \subset \Omega$ we define the boundary parts

$$
\partial_{\Omega} A:=\overline{\partial A \backslash \Sigma^{n}} \text { and } \partial_{\Sigma} A:=\partial A \backslash \partial_{\Omega} A .
$$

Now, the aim is to find a function $u: \Omega \rightarrow \mathbb{R}$ such that the hypersurface $M_{t}^{n}$ is given as the $t$-level set of $u$, i.e. $M_{t}^{n}=\partial_{\Omega}\{u<t\}$. The conditions on $u$ are described by the following degenerate elliptic mixed boundary value problem for $u$ :

$(\star)$

$$
\left\{\begin{aligned}
\operatorname{div}\left(\frac{D u}{|D u|}\right) & =|D u| & & \text { in } \Omega_{0}:=\Omega \backslash \overline{E_{0}}, \\
D_{\mu} u & =0 & & \text { on } \Sigma_{0}:=\partial_{\Sigma} \Omega_{0}, \\
u & =0 & & \text { on } \partial_{\Omega} E_{0},
\end{aligned}\right.
$$

where $E_{0}:=\{u<0\}$ and $\partial_{\Omega} E_{0}$ is supposed to be a $C^{2, \alpha}$-hypersurface which meets $\Sigma^{n}$ orthogonally.

Note that as long as $H\left(M_{t}^{n}\right)>0$ we have $M_{t}^{n}=\{u=t\},|D u|_{u=t} \mid=H\left(M_{t}^{n}\right)>0$ and $(\star)$ is equivalent to (IMCF). The purpose of this paper is to prove the existence and uniqueness of weak solutions of $(\star)$ in the sense of Definition 4.1.

Remark 2.2. (1) For $F_{0} \in C^{2, \alpha}\left(M^{n}\right)$ there exists a constant $T>0$ such that (IMCF) has a unique solution $F \in C^{2+\alpha, 1+\frac{\alpha}{2}}\left(M^{n} \times[0, T]\right) \cap C^{\infty}\left(M^{n} \times(0, T]\right)$. This also holds in a Riemannian ambient space [22].

(2) The corresponding Neumann problem for mean curvature flow was first studied by Stahl [30,31]. It was followed by the work of Buckland [3] who analyzed the singularities and by the work of Koeller [14,15] who proved further regularity results. Recently, Vulcanov [35] studied a mixed Dirichlet-Neumann boundary value problem for mean curvature flow.

(3) If $\Sigma^{n}$ is a convex cone and $M_{0}^{n}$ is a $C^{2, \alpha}$-hypersurface with strictly positive mean curvature which is star-shaped with respect to the center of the cone, then (IMCF) has a unique solution for all time. Furthermore, after suitable rescaling the hypersurfaces converge to a piece of a round sphere [23]. For closed hypersurfaces this result goes back to Gerhardt [4] (see also Urbas [33]).

(4) The definition of weak solutions of IMCF for closed hypersurfaces via elliptic regularization of $(\star)$ together with a variational approach goes back to Huisken and Ilmanen [11]. They proved the existence and uniqueness of those weak solutions which enabled them to prove the Riemannian Penrose inequality.

(5) Moser [25] showed that weak solutions in the sense of Huisken-Ilmanen can also be obtained by regularizing $(\star)$ with the help of $p$-harmonic functions. Later, Kotschwar and $\mathrm{Ni}$ [16] extended this result to Riemannian ambient spaces satisfying a volume growth condition. 
(6) Another related problem was considered by Hein [10] who proved the existence of weak solutions similar to those of Huisken-Ilmanen for a parabolic version of the level-set equation. This approach is also interesting with regard to a numerical treatment of IMCF.

(7) Schulze [27] applied the level-set approach to study flows with speed equal to positive powers of the mean curvature. In [28], he used this formulation to give a new proof of the isoperimetric inequality. Currently, Volkmann [34] is using the level-set approach to study the Neumann problem for flows with speed equal to positive powers of the mean curvature.

In order to solve $(\star)$ we want to consider a family of non-degenerate problems in bounded domains. It turns out that we also have to deform the given set $E_{0}$ in order to be able to solve the non-degenerate problem in the right weighted Hölder spaces.

Definition 2.3. Let $E_{0} \subset \Omega$ be relatively open in $\Omega$. Suppose $\partial_{\Omega} E_{0}$ is a $C^{2, \alpha}$-hypersurface which meets $\Sigma^{n}$ orthogonally. The approximating inner Dirichlet boundary $\partial_{\Omega} E_{0, \varepsilon}$ is defined with the help of the set

$$
E_{0, \varepsilon}:=E_{0} \backslash\left\{x \in E_{0} \mid \operatorname{dist}\left(x, \Sigma^{n}\right)<\varepsilon, \operatorname{dist}\left(x, \partial E_{0}\right)<\xi_{\varepsilon}(x)\right\}
$$

where

$$
\xi_{\varepsilon}(x):=\varepsilon^{3} \exp \left(1-\left(\frac{\varepsilon}{\varepsilon-\operatorname{dist}\left(x, \Sigma^{n}\right)}\right)^{2}\right) .
$$

Note that $\xi_{\varepsilon}$ is arranged such that

$$
0<D_{\mu} \operatorname{dist}\left(\cdot, E_{0, \varepsilon}\right)<1 \quad \text { on } \Sigma^{n} \cap \partial_{\Omega} E_{0, \varepsilon} .
$$

To define a family of approximating problems in bounded domains we also have to introduce an artificial outer Dirichlet boundary. If $F_{L_{\varepsilon}} \subset \Omega$ is open and $\partial_{\Omega} F_{L_{\varepsilon}}$ is a $C^{2, \alpha}$-hypersurface such that $F_{L_{\varepsilon}} \supset E_{0, \varepsilon}$ and

$$
-1<D_{\mu} \operatorname{dist}\left(\cdot, F_{L_{\varepsilon}}\right)<0 \text { on } \Sigma^{n} \cap \partial_{\Omega} F_{L_{\varepsilon}},
$$

then $\partial_{\Omega} F_{L_{\varepsilon}}$ is a suitable outer Dirichlet boundary. Finally, we also regularize the differential operator by defining

$$
Q^{\varepsilon} u:=\operatorname{div}\left(\frac{D u}{\sqrt{\varepsilon^{2}+|D u|^{2}}}\right)-\sqrt{\varepsilon^{2}+|D u|^{2}} .
$$

Using these notations we can formulate a family of $\varepsilon$-regularized level-set problems in bounded domains: For $\varepsilon>0$ we consider the family of regularized problems

$(\star)_{\varepsilon, \tau} \quad\left\{\begin{aligned} Q^{\varepsilon} u^{\varepsilon, \tau}=0 & \text { in } \Omega_{\varepsilon}:=F_{L_{\varepsilon}} \backslash \overline{E_{0, \varepsilon}}, \\ D_{\mu} u^{\varepsilon, \tau}=0 & \text { on } \Sigma_{\varepsilon}:=\partial_{\Sigma} \Omega_{\varepsilon}, \\ u^{\varepsilon, \tau}=0 & \text { on } \partial_{\Omega} E_{0, \varepsilon}, \\ u^{\varepsilon, \tau}=\tau & \text { on } \partial_{\Omega} F_{L_{\varepsilon}},\end{aligned}\right.$

where $\tau \in\left[0, L_{\varepsilon}\right]$ and $L_{\varepsilon}>0$. 


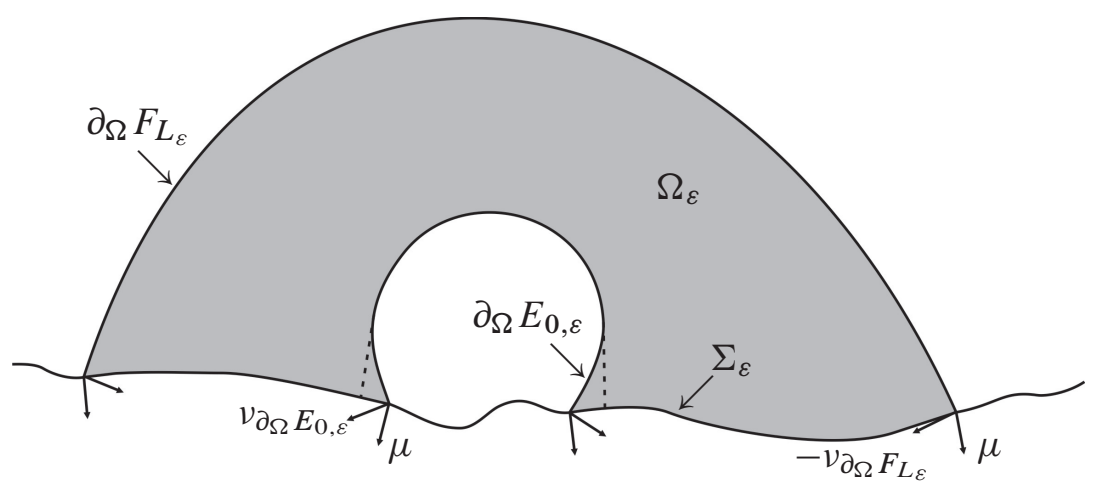

Figure 1. Domain and boundaries for $(\star)_{\varepsilon, \tau}$. The dotted line denotes $\partial_{\Omega} E_{0}$.

Remark 2.4. The aim is that for $\varepsilon \rightarrow 0$ the sets $F_{L_{\varepsilon}}$ become larger, $\partial_{\Omega} E_{0, \varepsilon}$ deforms back to $\partial_{\Omega} E_{0}$ and $L_{\varepsilon} \rightarrow \infty$ in order to recover the problem $(\star)$ in the limit. The existence of a solution of $(\star)_{\varepsilon, \tau}$ will depend on the choice of $F_{L_{\varepsilon}}$ and $L_{\varepsilon}$ as we will see in the next section. Furthermore, properties (2.1) and (2.2) ensure the existence of more regular solutions.

\section{Existence for the approximating problems}

We will prove the existence of solutions $u^{\varepsilon, \tau}$ of $(\star)_{\varepsilon, \tau}$ in weighted Hölder spaces. In particular, those solutions will satisfy $u^{\varepsilon, \tau} \in C^{2, \alpha}\left(\Omega_{\varepsilon}\right) \cap C^{1, \beta}\left(\overline{\Omega_{\varepsilon}}\right)$ for some $\alpha, \beta \in(0,1)$. This motivates the following definition:

Definition 3.1. Let $\alpha, \beta \in(0,1)$. A solution of $(\star)_{\varepsilon, \tau}$ with regularity

$$
u^{\varepsilon, \tau} \in C^{2, \alpha}\left(\Omega_{\varepsilon}\right) \cap C^{1, \beta}\left(\overline{\Omega_{\varepsilon}}\right)
$$

is called admissible. Furthermore, a function $u^{-}$with that regularity is a subsolution of $(\star)_{\varepsilon, \tau}$ if the following inequalities hold:

$$
\begin{aligned}
Q^{\varepsilon} u^{-} \geq 0 & \text { in } \Omega_{\varepsilon}, \\
D_{\mu} u^{-} \leq 0 & \text { on } \Sigma_{\varepsilon}, \\
u^{-} \leq 0 & \text { on } \partial_{\Omega} E_{0, \varepsilon}, \\
u^{-} \leq \tau & \text { on } \partial_{\Omega} F_{L_{\varepsilon}} .
\end{aligned}
$$

Similarly, a function $u^{+}$which satisfies the reverse inequalities it is called supersolution.

To obtain an estimate for $\left|u^{\varepsilon, \tau}\right|$ we first note that $u_{\tau}^{+}: \equiv \tau$ is a supersolution of $(\star)_{\varepsilon, \tau}$ and thus $u^{\varepsilon, \tau}<\tau$. Unfortunately, constant functions do not serve as a subsolution. If we want to find a non-constant subsolution $u^{-}$with the help of the maximum principle, the sign of the quantity $D_{\mu} u^{-}$has to be controlled everywhere on $\Sigma_{\varepsilon}$. To achieve this we restrict to the case where $\Sigma^{n}$ is globally given as the graph of a $C^{1}$-function $f: \mathbb{R}^{n+1} \rightarrow \mathbb{R}$ such that all tangent lines to graph $f$ in radial directions hit the $x^{n+1}$-axis above the point $x_{0}:=\left(0, \ldots, 0,-c_{0}\right)$, i.e.

$$
\min _{x \in \mathbb{R}^{n+1}}\{f(x)-\langle x, D f(x)\rangle\}>-c_{0}
$$

for some $c_{0}>0$ sufficiently large. With the help of this condition we obtain a lower bound. 
Lemma 3.2 (Existence of a subsolution). Let $n \geq 2$. Let $\Sigma^{n}$ be globally given as the graph of a $C^{1}$-function $f: \mathbb{R}^{n+1} \rightarrow \mathbb{R}$ such that (3.1) holds. We set

$$
a:=\max _{\partial_{\Omega} E_{0, \varepsilon}} \operatorname{dist}\left(\cdot, x_{0}\right), \quad \varepsilon<\frac{1}{4 a}, \quad R:=\frac{1}{4 \varepsilon}, \quad 0 \leq \tau \leq L_{\varepsilon}:=\frac{1}{2} \ln \left(\frac{R}{a}\right)
$$

and define

$$
F_{L_{\varepsilon}}:=\left\{x \in \Omega \mid \operatorname{dist}\left(x, x_{0}\right)<R\right\} .
$$

Then an admissible solution $u^{\varepsilon, \tau}$ of $(\star)_{\varepsilon, \tau}$ satisfies the estimate

$$
u^{\varepsilon, \tau}(x) \geq u_{\tau}^{-}(x):=\frac{1}{2} \ln \left(\frac{\operatorname{dist}\left(x, x_{0}\right)}{a}\right)-L_{\varepsilon}+\tau .
$$

Proof. To obtain a subsolution of $(\star)_{\varepsilon, L_{\varepsilon}}$ we make the ansatz

$$
u^{-}(x):=\lambda \ln \left(\frac{r(x)}{a}\right), \quad L_{\varepsilon}:=\lambda \ln \left(\frac{R}{a}\right), \quad \lambda>0, \quad R>0
$$

where $r(x):=\operatorname{dist}\left(x, x_{0}\right)$ and $\lambda$ and $R$ will be specified later. In order to apply the maximum principle ${ }^{2)}$ we note that

$$
\begin{array}{ll}
u^{-} \leq 0 & \text { on } \partial_{\Omega} E_{0, \varepsilon}, \\
u^{-}=L_{\varepsilon} & \text { on } \partial_{\Omega} F_{L_{\varepsilon}} .
\end{array}
$$

Using (3.1) we obtain

$$
D_{\mu(x)} u^{-}(x)=\left\langle\mu(x), D u^{-}(x)\right\rangle=\frac{\lambda}{r^{2}(x)}\left\langle\mu(x), x-x_{0}\right\rangle \leq 0
$$

for $x \in \Sigma_{\varepsilon}$. It is left to prove the inequality for the operator $Q^{\varepsilon}$. We compute

$$
Q^{\varepsilon}\left(u^{-}\right) \geq \frac{1}{r\left(\varepsilon^{2} r^{2}+\lambda^{2}\right)^{3 / 2}}\left(-\lambda^{4}+\lambda^{3}-2 \varepsilon^{2} r^{2} \lambda^{2}-\varepsilon^{4} r^{4}\right)
$$

provided $n \geq 2$. Thus, if we choose $\lambda=1 / 2, r \leq R:=1 / 4 \varepsilon$ and $\varepsilon<1 / 4 a$, then $Q^{\varepsilon}\left(u^{-}\right)>0$ and the maximum principle implies $u^{-} \leq u^{\varepsilon, \bar{L}_{\varepsilon}}$ in $\overline{\Omega_{\varepsilon}}$. So far we obtained a subsolution of $(\star)_{\varepsilon, \tau=L_{\varepsilon}}$, so we rename $u^{-}$to $u_{L_{\varepsilon}}^{-}$and note that the function $u_{\tau}^{-}:=u_{L_{\varepsilon}}^{-}-L_{\varepsilon}+\tau$ is a subsolution of $(\star)_{\varepsilon, \tau}$.

Note that the estimate $u^{\varepsilon, \tau} \geq u_{\tau}^{-}$is not very accurate near $\partial_{\Omega} E_{0, \varepsilon}$ since it does not imply that $u^{\varepsilon, \tau}$ becomes non-negative as $\varepsilon$ tends to zero. Using subsolutions which are less steep we can fix this problem.

Lemma 3.3 (Improved lower bound). Let $n, a, \Sigma^{n}, F_{L_{\varepsilon}}, L_{\varepsilon}$ be defined as in Lemma 3.2. If $\varepsilon>0$ is sufficiently small and $R$ is defined as $R:=1 /\left(10 \varepsilon^{1 / 64}\right)$, then an admissible solution $u^{\varepsilon, \tau}$ of $(\star)_{\varepsilon, \tau}$ satisfies

$$
u^{\varepsilon, \tau} \geq \max \left\{-\varepsilon^{5 / 4}, u_{\tau}^{-}\right\}
$$

2) Here and in the following we use a version of the comparison principle for quasilinear elliptic operators similar to [6, Theorem 10.1]. 
Proof. We define a new subsolution of $(\star)_{\varepsilon, \tau}$ by

$$
v^{-}(x):=\eta u_{\tau}^{-}(x)=\eta\left(\frac{1}{2} \ln \left(\frac{r(x)}{a}\right)-L_{\varepsilon}+\tau\right)
$$

with $r(x):=\operatorname{dist}\left(x, x_{0}\right)$. We see that for $\eta \in[0,1]$ the function $v^{-}=\eta u^{-}$satisfies the right inequalities at the boundary

$$
\left.v^{-}\right|_{\partial_{\Omega} E_{0, \varepsilon}} \leq 0,\left.\quad v^{-}\right|_{\partial_{\Omega}} F_{L_{\varepsilon}} \leq \tau,\left.\quad D_{\mu} v^{-}\right|_{\Sigma_{\varepsilon}} \leq 0 .
$$

Similar to the proof of Lemma 3.2 we compute $Q^{\varepsilon}\left(v^{-}\right)$. For $n \geq 2, \varepsilon>0$ sufficiently small, $\eta:=\varepsilon^{21 / 16}$ and $r \leq 1 /\left(10 \varepsilon^{1 / 64}\right)=: R$ we obtain $Q^{\varepsilon}\left(v^{-}\right) \geq 0$. Thus, the maximum principle implies

$$
u^{\varepsilon, \tau}(x) \geq v^{-}(x) \geq-\varepsilon^{5 / 4} \varepsilon^{1 / 16}\left(C+\left|\ln \left(\varepsilon^{1 / 16}\right)\right|\right) \geq-\varepsilon^{5 / 4}
$$

for $\varepsilon \leq C^{-16}$ where $C=C\left(a, \min _{\Omega_{\varepsilon}} \operatorname{dist}\left(\cdot, x_{0}\right)\right)$.

Now we estimate the gradient. We start with the estimates on the Dirichlet boundary parts $\partial_{\Omega} E_{0, \varepsilon}$ and $\partial_{\Omega} F_{L_{\varepsilon}}$. On $\partial_{\Omega} F_{L_{\varepsilon}}$ we can directly use super- and subsolutions $u_{\tau}^{+}$and $u_{\tau}^{-}$ as barriers.

Lemma 3.4 (Gradient estimate on $\partial_{\Omega} F_{L_{\varepsilon}}$ ). Assume that there exists an admissible subsolution $u_{L_{\varepsilon}}$ of $(\star)_{\varepsilon, L_{\varepsilon}}$ such that $F_{L_{\varepsilon}}:=\left\{u_{L_{\varepsilon}}^{-}<L_{\varepsilon}\right\}$. Then an admissible solution $u^{\varepsilon, \tau}$ of $(\star)_{\varepsilon, \tau}$ satisfies the gradient estimate

$$
0 \leq D_{\nu} u^{\varepsilon, \tau} \leq D_{\nu} u_{L_{\varepsilon}} \text { on } \partial_{\Omega} F_{L_{\varepsilon}}
$$

where $v$ is the exterior unit normal to $\partial_{\Omega} F_{L_{\varepsilon}}$ with respect to the set $F_{L_{\varepsilon}}$. Under the hypothesis of Lemma 3.3 we obtain the more explicit estimate $D_{\nu} u^{\varepsilon, \tau} \leq 5 \varepsilon^{1 / 64}$.

Proof. The lower bound follows from the fact that $u_{\tau}^{+}:=\tau$ is a supersolution of $(\star)_{\varepsilon, \tau}$ which coincides with $u^{\varepsilon, \tau}$ on $\partial_{\Omega} F_{L_{\varepsilon}}$. Similarly, $u_{\tau}^{-}:=u_{L_{\varepsilon}}^{-}-L_{\varepsilon}+\tau$ is a subsolution of $(\star)_{\varepsilon, \tau}$ which coincides with $u^{\varepsilon, \tau}$ on $\partial_{\Omega} F_{L_{\varepsilon}}$. This yields the upper bound. Under the hypothesis of Lemma 3.3 we obtain an explicit subsolution $u_{\tau}^{-}$which yields the estimate

$$
D_{\nu} u^{\varepsilon, \tau} \leq 5 \varepsilon^{1 / 64}
$$

In order to estimate the gradient on $\partial_{\Omega} E_{0, \varepsilon}$ we construct barriers $\rho$ having a product structure which involves the distance to $\partial_{\Omega} E_{0, \varepsilon}$ and $\Sigma_{\varepsilon}$. In a first step we compute $Q^{\varepsilon}(\rho)$ and $D_{\mu} \rho$ for this type of barriers.

Lemma 3.5 (Formulas for barriers having a product structure). Let $d:=\operatorname{dist}_{\partial_{\Omega}} E_{0, \varepsilon}$ and $s:=\operatorname{dist}_{\Sigma_{\varepsilon}}$, and assume that the distance functions are evaluated in a region where they are $C^{2}$. Let $f, g \in C^{2}(\mathbb{R})$. Then a barrier of the form $\rho(x):=f(d(x)) \cdot g(s(x))$ satisfies

$$
\left(\left|f^{\prime} g\right|-\left|f g^{\prime}\right|\right)^{2} \leq|D \rho|^{2} \leq\left(\left|f^{\prime} g\right|+\left|f g^{\prime}\right|\right)^{2} .
$$

The Neumann condition reads

$$
\left.D_{\mu(x)} \rho(x)\right|_{\Sigma_{\varepsilon}}=\left.f^{\prime}(d(x))\right|_{\Sigma_{\varepsilon}} g(0) D_{\mu(x)} d(x)\left|\Sigma_{\varepsilon}-f(d(x))\right|{ }_{\Sigma_{\varepsilon}} g^{\prime}(0)
$$


and for the differential operator $Q^{\varepsilon}$ we obtain

$$
\begin{aligned}
\sqrt{\varepsilon^{2}+|D \rho|^{2}} Q^{\varepsilon} \rho=f^{\prime} & g\left(\delta^{i j}-\frac{f^{2} g^{\prime 2} D^{i} s D^{j} s}{\varepsilon^{2}+|D \rho|^{2}}\right) D_{i j} d \\
& +f g^{\prime}\left(\delta^{i j}-\frac{f^{\prime 2} g^{2} D^{i} d D^{j} d}{\varepsilon^{2}+|D \rho|^{2}}\right) D_{i j} s-\varepsilon^{2}-|D \rho|^{2} \\
& +\frac{f^{\prime \prime} g}{\varepsilon^{2}+|D \rho|^{2}}\left(\varepsilon^{2}+f^{2} g^{\prime 2}\left(1-\langle D d, D s\rangle^{2}\right)\right) \\
& +\frac{f g^{\prime \prime}}{\varepsilon^{2}+|D \rho|^{2}}\left(\varepsilon^{2}+f^{\prime 2} g^{2}\left(1-\langle D d, D s\rangle^{2}\right)\right) \\
& +\frac{2 f^{\prime} g^{\prime}}{\varepsilon^{2}+|D \rho|^{2}}\left(\varepsilon^{2}\langle D d, D s\rangle+f f^{\prime} g g^{\prime}\left(\langle D d, D s\rangle^{2}-1\right)\right) .
\end{aligned}
$$

Proof. The formulas follow from a direct calculation using the fact that $D s=-\mu$ and $|D d|=1=|D s|$.

Remark 3.6. Note that in general $\partial_{\Omega} E_{0, \varepsilon}$ has to be extended below $\Sigma^{n}$ in a small neighborhood of $\partial_{\Omega} E_{0} \cap \Sigma^{n}$ in order to use the distance function in a neighborhood of the corner. This extension can be constructed to have the same $C^{2}$-norm as $\partial_{\Omega} E_{0, \varepsilon}$ so the estimates are independent of this extension.

First, we use the product ansatz to construct a barrier on $\partial_{\Omega} E_{0, \varepsilon}$ from below.

Lemma 3.7 (Gradient estimate on $\partial_{\Omega} E_{0, \varepsilon}$ from below). Let $\varepsilon>0$ be sufficiently small. Suppose that $\partial_{\Omega} E_{0, \varepsilon}$ and $\Sigma_{\varepsilon}$ are $C^{2}$-hypersurfaces. If $u^{\varepsilon, \tau}$ is an admissible solution of $(\star)_{\varepsilon, \tau}$ which satisfies $u^{\varepsilon, \tau} \geq-\varepsilon^{1+\gamma}$ for some $\gamma \in(0,1)$, then the following gradient estimate holds:

$$
D_{\nu} u^{\varepsilon, \tau} \geq-2 \varepsilon \quad \text { on } \partial_{\Omega} E_{0, \varepsilon}
$$

where $v$ is the exterior unit normal to $\partial_{\Omega} E_{0, \varepsilon}$ with respect to the set $E_{0, \varepsilon}$.

Proof. Let $d:=\operatorname{dist}_{\partial_{\Omega}} E_{0, \varepsilon}$ and $s:=\operatorname{dist}_{\Sigma_{\varepsilon}}$. We restrict ourselves to the set

$$
\Gamma:=\left\{x \in \Omega_{\varepsilon} \mid d(x)<d_{\max }\right\} .
$$

The value of $d_{\max }>0$ will be determined later. The boundary of $\Gamma$ consists of $\partial_{\Omega} E_{0, \varepsilon}, \partial_{\Sigma} \Gamma$ and a new boundary part in the interior of $\Omega_{\varepsilon}$ which we call $\partial \Gamma_{1}$. We make the ansatz

$$
\rho(x):=f(d(x)) \cdot g(s(x))
$$

with

$$
f(d):=\frac{\varepsilon}{A}(\exp (-A d)-1)
$$

and see that $f, f^{\prime}$ and $f^{\prime \prime}$ satisfy

$$
-\frac{\varepsilon}{A} \leq f \leq 0, \quad-\varepsilon \leq f^{\prime} \leq-\frac{\varepsilon}{2}, \quad \frac{\varepsilon A}{2} \leq f^{\prime \prime} \leq \varepsilon A
$$

where the upper bound on $f^{\prime}$ and the lower bound on $f^{\prime \prime}$ require $d_{\max } \leq \ln (2) / A$. For $g$ we choose

$$
g(s):= \begin{cases}1+\exp \left(2-2\left(\frac{s_{\max }}{s_{\max }-s}\right)^{2}\right) & \text { for } 0 \leq s<s_{\max } \\ 1 & \text { for } s \geq s_{\max },\end{cases}
$$


and a direct calculation shows that

$$
1 \leq g \leq 2, \quad-\frac{4}{s_{\max }} \leq g^{\prime} \leq 0, \quad 0 \leq g^{\prime \prime} \leq \frac{12}{s_{\max }^{2}}
$$

The exact values $d_{\max }, s_{\max }$ and $A$ will be determined later. We see that $\rho$ is a negative function which satisfies the Dirichlet boundary condition $\rho=0$ on $\partial_{\Omega} E_{0, \varepsilon}$ since $f(0)=0$. Using $u^{\varepsilon, \tau} \geq-\varepsilon^{1+\gamma}$ we see that

$$
\rho \leq-\frac{\varepsilon}{A}\left(1-\exp \left(-A d_{\max }\right)\right) \leq u^{\varepsilon, \tau}
$$

on $\partial \Gamma_{1}$ for $\varepsilon>0$ sufficiently small. Next we want to verify that $D_{\mu} \rho \leq 0$ on $\partial_{\Sigma} \Gamma$. By construction of $E_{0, \varepsilon}$ we know that (2.1) holds. Thus, there is some $C_{1}>0$ such that $D_{\mu} d \geq-C_{1} d$ on $\partial_{\Sigma} \Gamma$. Furthermore, we note that $f(d) \leq-\varepsilon d / 2$ for $d_{\max } \leq A^{-1}$. Using these estimates together with (3.3) and (3.5) yields

$$
D_{\mu} \rho=2 f^{\prime}(d) D_{\mu} d+\frac{4}{d_{\max }} f(d) \leq 2 \varepsilon\left(C_{1}-\frac{1}{d_{\max }}\right) d \leq 0
$$

on $\partial_{\Sigma} \Gamma$ for $d_{\max } \leq \min \left\{C_{1}^{-1}, A^{-1}\right\}$. Finally, we have to make sure that $Q^{\varepsilon}(\rho) \geq 0$. Using (3.4), (3.5) and (3.6) we obtain

$$
\begin{aligned}
\sqrt{\varepsilon^{2}+|D \rho|^{2}} Q^{\varepsilon} \rho \geq f^{\prime} g( & \left.\delta^{i j}-\frac{f^{2} g^{\prime 2} D^{i} s D^{j} s}{\varepsilon^{2}+|D \rho|^{2}}\right) D_{i j} d \\
& +f g^{\prime}\left(\delta^{i j}-\frac{f^{\prime 2} g^{2} D^{i} d D^{j} d}{\varepsilon^{2}+|D \rho|^{2}}\right) D_{i j} s-\varepsilon^{2}-|D \rho|^{2} \\
& +\frac{f^{\prime \prime} g}{\varepsilon^{2}+|D \rho|^{2}} \varepsilon^{2}+\frac{f g^{\prime \prime}}{\varepsilon^{2}+|D \rho|^{2}}\left(\varepsilon^{2}+f^{\prime 2} g^{2}\right)-\frac{2 f^{\prime} g^{\prime}}{\varepsilon^{2}+|D \rho|^{2}} \varepsilon^{2}
\end{aligned}
$$

where the only positive term is the one which involves $f^{\prime \prime}$. If we are further than $s_{\max }$ away from $\Sigma_{\varepsilon}$, the function $g \equiv 1,|D \rho|^{2}=\left(f^{\prime}\right)^{2}$ and the estimate reads

$$
\sqrt{\varepsilon^{2}+|D \rho|^{2}} Q^{\varepsilon} \rho=f^{\prime} \Delta d-\varepsilon^{2}-\left(f^{\prime}\right)^{2}+\frac{\varepsilon^{2}}{\varepsilon^{2}+\left(f^{\prime}\right)^{2}} f^{\prime \prime} \geq 0
$$

for $\varepsilon \leq 1$ and $A \geq 4\left(2+n^{2}\left|D^{2} d\right|\right)$. Before we continue with the estimate close to $\Sigma_{\varepsilon}$ we have to estimate $|D \rho|^{2}$. We use (3.2), (3.5), (3.6) and $A s_{\max } \geq 24$ to see that

$$
\frac{1}{9} \varepsilon^{2} \leq\left(\frac{\varepsilon}{2}-\frac{4 \varepsilon}{A s_{\max }}\right)^{2} \leq|D \rho|^{2} \leq\left(2 \varepsilon+\frac{4 \varepsilon}{A s_{\max }}\right)^{2} \leq 9 \varepsilon^{2} .
$$

This estimate together with (3.5) and (3.6) allows us to estimate

$$
\left|f^{\prime} g\left(\delta^{i j}-\frac{f^{2} g^{\prime 2} D^{i} s D^{j} s}{\varepsilon^{2}+|D \rho|^{2}}\right) \xi_{i} \xi_{j}\right| \leq 4 \varepsilon|\xi|^{2}
$$

and

$$
\left|f g^{\prime}\left(\delta^{i j}-\frac{f^{\prime 2} g^{2} D^{i} d D^{j} d}{\varepsilon^{2}+|D \rho|^{2}}\right) \xi_{i} \xi_{j}\right| \leq 7 \varepsilon|\xi|^{2}
$$


where we used again $A s_{\max } \geq 24$. Putting everything together we obtain an estimate for $Q^{\varepsilon} \rho$ away from $\Sigma_{\varepsilon}$

$$
\sqrt{\varepsilon^{2}+|D \rho|^{2}} Q^{\varepsilon} \rho \geq \frac{\varepsilon}{20 A s_{\max }^{2}}\left(\left(A s_{\max }\right)^{2}-C_{2}\left(A s_{\max }\right)-C_{2}\right) \geq 0
$$

for $\varepsilon \leq 1, s_{\max } \leq 1, C_{2}:=10000\left(n^{2}\left|D^{2} d\right|+n^{2}\left|D^{2} s\right|+1\right)$ and $A s_{\max } \geq 2 C_{2}$. Altogether we see that $\rho$ is a subsolution of $(\star)_{\varepsilon, \tau}$ in $\Gamma$ for $\varepsilon>0$ sufficiently small and the choice of parameters

$$
s_{\max }:=\eta, \quad A:=\frac{2 C_{2}}{s_{\max }}, \quad d_{\max }:=\min \left\{C_{1}^{-1}, A^{-1}, \eta\right\} .
$$

Using the maximum principle we conclude that

$$
D_{\nu} u^{\varepsilon, \tau} \geq D_{\nu} \rho=f^{\prime}(0) g D_{\nu} d+f(0) g^{\prime} D_{\nu} s=-\varepsilon g \geq-2 \varepsilon \quad \text { on } \partial_{\Omega} E_{0, \varepsilon}
$$

where $v$ is the exterior unit normal to $\partial_{\Omega} E_{0, \varepsilon}$ with respect to the set $E_{0, \varepsilon}$.

In the next step we construct a barrier on $\partial_{\Omega} E_{0, \varepsilon}$ from above.

Lemma 3.8 (Gradient estimate on $\partial_{\Omega} E_{0, \varepsilon}$ from above). Let $\varepsilon>0$ be sufficiently small. Suppose that $\partial_{\Omega} E_{0, \varepsilon}$ and $\Sigma_{\varepsilon}$ are $C^{2}$-hypersurfaces. If $u^{\varepsilon, \tau}$ is an admissible solution of $(\star)_{\varepsilon, \tau}$, then the following gradient estimate holds:

$$
D_{\nu} u^{\varepsilon, \tau} \leq C\left(n, \partial_{\Omega} E_{0}, \Sigma^{n}\right) \quad \text { on } \partial_{\Omega} E_{0, \varepsilon}
$$

where $v$ is the exterior unit normal to $\partial_{\Omega} E_{0, \varepsilon}$ with respect to the set $E_{0, \varepsilon}$.

Proof. Let $d, s, \Gamma, \rho, g$ be as in the proof of Lemma 3.7. Furthermore, let $f(d):=A d$ for some $A>0$. The exact values $d_{\max }, s_{\max }$ and $A$ will be determined later. We see that $\rho$ is a positive function which satisfies the Dirichlet boundary condition $\rho=0$ on $\partial_{\Omega} E_{0, \varepsilon}$ since $f(0)=0$. Furthermore, $\rho$ lies above $u^{\varepsilon, \tau}$ on $\partial \Gamma_{1}$ since

$$
\rho=f\left(d_{\max }\right) \cdot g(s(x)) \geq A d_{\max } \geq u^{\varepsilon, \tau}
$$

on $\partial \Gamma_{1}$ for $A d_{\max } \geq \tau$. To verify that $D_{\mu} \rho \geq 0$ on $\partial_{\Sigma} \Gamma$ we use (3.3) together with the estimate $D_{\mu} d \geq-C_{1} d$ on $\partial_{\Sigma} \Gamma$ to obtain

$$
D_{\mu} \rho=2 A D_{\mu} d+\frac{4}{d_{\max }} A d \geq 2 A\left(-C_{1}+\frac{2}{d_{\max }}\right) d \geq 0
$$

on $\partial_{\Sigma} \Gamma$ for $d_{\max } \leq 2 C_{1}^{-1}$. In contrast to Lemma 3.7 we first prove that $Q^{0} \rho \geq 0$. Using (3.4) and (3.6) we obtain

$$
\begin{gathered}
|D \rho| Q^{0} \rho \leq f^{\prime} g\left(\delta^{i j}-\frac{f^{2} g^{\prime 2} D^{i} s D^{j} s}{|D \rho|^{2}}\right) D_{i j} d+f g^{\prime}\left(\delta^{i j}-\frac{f^{\prime 2} g^{2} D^{i} d D^{j} d}{|D \rho|^{2}}\right) D_{i j} s \\
-|D \rho|^{2}+\frac{f g^{\prime \prime}}{|D \rho|^{2}} f^{\prime 2} g^{2} .
\end{gathered}
$$

Here the only good term is $-|D \rho|^{2}$. In the case that we are far from $\Sigma_{\varepsilon}$ we have $g \equiv 1$ and $|D \rho|^{2}=\left|f^{\prime}\right|^{2}$. Therefore, the estimate simplifies and we obtain

$$
|D \rho| Q^{0} \rho \leq f^{\prime} \Delta d-|D \rho|^{2} \leq A n^{2}\left|D^{2} d\right|-A^{2} \leq 0
$$


for $A \geq n^{2}\left|D^{2} d\right|$. As in Lemma 3.7 we proceed by estimating $|D \rho|^{2}$. We use (3.2) and (3.6) and choose $d_{\max }:=s_{\max } / 8$ to compute that

$$
\frac{A^{2}}{4} \leq A^{2}\left(1-\frac{4 d_{\max }}{s_{\max }}\right)^{2} \leq|D \rho|^{2} \leq 4 A^{2}\left(1+\frac{2 d_{\max }}{s_{\max }}\right)^{2} \leq 7 A^{2} .
$$

This yields

$$
\left|f^{\prime} g\left(\delta^{i j}-\frac{f^{2} g^{\prime 2} D^{i} s D^{j} s}{|D \rho|^{2}}\right) \xi_{i} \xi_{j}\right| \leq 6 A|\xi|^{2}
$$

and

$$
\left|f g^{\prime}\left(\delta^{i j}-\frac{f^{\prime 2} g^{2} D^{i} d D^{j} d}{|D \rho|^{2}}\right) \xi_{i} \xi_{j}\right| \leq 9 A|\xi|^{2} .
$$

Combining these estimates we obtain

$$
|D \rho| Q^{0} \rho \leq \frac{A}{4}\left(1000\left(n^{2}\left|D^{2} d\right|+n^{2}\left|D^{2} s\right|+s_{\max }^{-1}\right)-A\right) \leq 0
$$

for $A \geq 1000\left(n^{2}\left|D^{2} d\right|+n^{2}\left|D^{2} s\right|+s_{\max }^{-1}\right)=: 1000\left(C_{3}+s_{\max }^{-1}\right)$. To summarize, we proved that $\rho$ is a supersolution of $(\star)_{0, \tau}$ in $\Gamma$ for the parameters

$$
d_{\max }:=\min \left\{2 C_{1}^{-1}, \eta\right\}, \quad s_{\max }:=8 d_{\max }, \quad A_{\tau}:=1000\left(C_{3}+s_{\max }^{-1}\right)+\frac{\tau}{d_{\max }} .
$$

So far, to match increasing boundary values $\tau$ on $\partial \Gamma_{1}$ we have to choose steeper functions $\rho$. This means that in the limit $\varepsilon \rightarrow 0\left(L_{\varepsilon} \rightarrow \infty\right)$ we loose the gradient estimate. To prevent this from happening we choose the function $\rho$ corresponding to $\tau:=1$ and consider the subdomain $\tilde{\Gamma}:=\{0 \leq \rho<1\} \subset \Gamma$. On $\tilde{\Gamma}$ we define

$$
\tilde{\rho}:=\frac{\rho}{1-\rho} .
$$

We see that $\tilde{\rho}=0$ on $\partial_{\Omega} E_{0, \varepsilon}$ since $\rho=0$ on $\partial_{\Omega} E_{0, \varepsilon}$. Furthermore, we obtain the same sign for $D_{\mu} \tilde{\rho}$ as for $D_{\mu} \rho$. The PDE is also satisfied with the same inequality since

$$
Q^{0} \tilde{\rho} \leq Q^{0} \rho \leq-\frac{A_{1}}{4|D \rho|}\left(1000\left(C_{3}+s_{\max }^{-1}\right)-A_{1}\right) \leq-\frac{1}{12 d_{\max }} .
$$

In contrast to $\rho$ the function $\tilde{\rho}$ is a supersolution of $(\star)_{\varepsilon, \tau}$ on $\{0 \leq \tilde{\rho} \leq \tau\} \subset \tilde{\Gamma}$ for arbitrary large boundary values since the function blows up when it approaches the boundary $\{\rho=1\}$. Next, we observe that

$$
\left|Q^{\varepsilon} \tilde{\rho}-Q^{0} \tilde{\rho}\right| \leq\left(1+\frac{3\left|D^{2} \tilde{\rho}\right|}{|D \tilde{\rho}|^{2}}\right) \varepsilon \leq c_{1} \varepsilon
$$

Therefore, by continuity we know that $Q^{\varepsilon} \tilde{\rho}<0$ for $\varepsilon$ sufficiently small. Thus, $\tilde{\rho}$ is also a supersolution of $(\star)_{\varepsilon, \tau}$ for $\varepsilon>0$ sufficiently small and arbitrary $\tau$. This yields the estimate

$$
D_{\nu} u \leq D_{\nu} \tilde{\rho}=D_{\nu} \rho=C\left(n, \partial E_{0}, \Sigma^{n}\right)
$$

on $\partial_{\Omega} E_{0, \varepsilon}$. Note that we can estimate the $C^{2}$-norm of $d$ independently of the approximation of $\partial_{\Omega} E_{0}$ by $\partial_{\Omega} E_{0, \varepsilon}$. Thus, the estimate is independent of $\varepsilon$.

The remaining boundary part of the domain $\Omega_{\varepsilon}$ is the Neumann boundary part $\Sigma_{\varepsilon}$. If the supporting hypersurface is convex, the maximum principle tells us that a maximum of the gradient cannot occur on $\Sigma_{\varepsilon}$. 
Lemma 3.9 (Gradient estimate on $\Sigma_{\varepsilon}$ ). Let $\Sigma_{\varepsilon}$ be a convex $C^{3}$-hypersurface. Let $u^{\varepsilon, \tau}$ be an admissible solution of $(\star)_{\varepsilon, \tau}$. Then, $\left|D u^{\varepsilon, \tau}\right|$ cannot attain a maximum on $\Sigma_{\varepsilon}$.

Proof. Let $x_{0} \in \Sigma_{\varepsilon}$. First, we note that due to the regularity of $\Sigma_{\varepsilon}$ there is a neighborhood of $x_{0}$ in $\Omega_{\varepsilon}$ in which $u:=u^{\varepsilon, \tau}$ is $C^{3}$. Let us define $v:=|D u|^{2} / 2$. Let

$$
a^{i}(p):=\frac{p^{i}}{\sqrt{\varepsilon^{2}+|p|^{2}}} \quad \text { and } \quad a^{i j}(p):=\frac{\partial a^{i}(p)}{\partial p^{j}} \text {. }
$$

We apply the operator $\left(D^{j} u\right) D_{j}$ to $Q^{\varepsilon}(u)$ defined in $(\star)_{\varepsilon, \tau}$. Here $j$ runs from 1 to $n$. This yields

$$
\begin{aligned}
L v & :=D_{i}\left(a^{i k}(D u) D_{k} v\right)-\frac{D^{j} u}{\sqrt{\varepsilon^{2}+|D u|^{2}}} D_{j} v \\
& \geq D_{i}\left(a^{i k}(D u) D^{j} u D_{k j} u\right)-a^{i k}(D u) D_{i}^{j} u D_{k j} u-\frac{D^{j} u}{\sqrt{\varepsilon^{2}+|D u|^{2}}} D^{k} u D_{k j} u \\
& =D^{j} u D_{j} \operatorname{div}\left(\frac{D u}{\sqrt{\varepsilon^{2}+|D u|^{2}}}\right)-D^{j} u D_{j} \sqrt{\varepsilon^{2}+|D u|^{2}}=0 .
\end{aligned}
$$

Assume that the maximum of $v$ is attained at $x_{0}$. In a neighborhood of $x_{0}$ we choose an orthonormal frame such that $e_{1}, \ldots, e_{n-1} \in T_{x_{0}} \Sigma_{\varepsilon}$ and $e_{n}=\mu$. At $x_{0}$ we have

$$
\begin{aligned}
D_{\mu} v & =\sum_{i=1}^{n-1} D_{e_{i}} u\left(D_{e_{i}}\left(D_{e_{n}} u\right)-\sum_{j=1}^{n-1}\left\langle D_{e_{i}} e_{n}, e_{j}\right\rangle D_{e_{j}} u\right) \\
& =-\sum_{i, j=1}^{n-1} \Sigma_{\varepsilon} h\left(e_{i}, e_{j}\right) D_{e_{i}} u D_{e_{j}} u \leq 0
\end{aligned}
$$

since $\Sigma_{\varepsilon}$ is convex. The signs for $D_{\mu} v$ and $L v$ together with the maximum principle imply that $v$ cannot attain a maximum on $\Sigma_{\varepsilon}$.

Remark 3.10. Using the maximum principle, inequality (3.7) implies that the gradient of an admissible solution of $(\star)_{\varepsilon, \tau}$ cannot attain a maximum in the interior of $\Omega_{\varepsilon}$.

All together we obtain the following a priori estimates for $|u|,|D u|$ and the weighted Hölder norm $\|\cdot\|_{2, \alpha ; \Omega_{\varepsilon}}^{(-1-\beta)}$ which is defined in Definition A.1 in the appendix.

Proposition 3.11. Assume that an admissible subsolution $u_{L_{\varepsilon}}$ of $(\star)_{\varepsilon, L_{\varepsilon}}$ exists such that $F_{L_{\varepsilon}}=\left\{u_{L_{\varepsilon}}<L_{\varepsilon}\right\}$ satisfies condition (2.2). Let $u$ be an admissible solution of $(\star)_{\varepsilon, \tau}$ such that $u \geq-\varepsilon^{1+\gamma}$ for some $\gamma \in(0,1)$ and that $|D u|_{\Sigma_{\varepsilon}} \leq c_{1}$ independently of $\varepsilon$. Then, for $\varepsilon>0$ sufficiently small

$$
-\varepsilon^{1+\gamma} \leq u \leq \tau \quad \text { and } \quad|D u| \leq C\left(n, \partial_{\Omega} E_{0}, \Sigma^{n}, c_{1},\left|D u_{L_{\varepsilon}}^{-}\right|\right)
$$

on $\Omega_{\varepsilon}$. Furthermore,

$$
\|u\|_{2, \alpha, \Omega_{\varepsilon}}^{(-1-\beta)} \leq C\left(n, \varepsilon, \partial_{\Omega} E_{0, \varepsilon}, \Sigma^{n}, L_{\varepsilon},\left|D u_{L_{\varepsilon}}^{-}\right|\right)
$$

for some $\beta=\beta\left(D_{\mu} \operatorname{dist}_{E_{0, \varepsilon}}, D_{\mu} \operatorname{dist}_{F_{L \varepsilon}}\right) \in(0,1)$. 
Proof. The estimates for $|u|$ and $|D u|$ follow from our previous lemmas in conjunction with the existence of a subsolution which satisfies $u \geq-\varepsilon^{1+\gamma}$. Furthermore, the gradient estimate tells us that the elliptic equation in $(\star)_{\varepsilon, \tau}$ which is equivalent to

$$
a^{i j}(D u) D_{i j} u:=\frac{1}{\varepsilon^{2}+|D u|^{2}}\left(\delta^{i j}-\frac{D^{i} u D^{j} u}{\varepsilon^{2}+|D u|^{2}}\right) D_{i j} u=1
$$

can be regarded as a linear, uniformly elliptic equation with bounded coefficients and righthand side. Therefore, [17, Chapter 2, Section 6, Theorem 6.1] implies that

$$
D u \in H_{0, \alpha}^{(0)}\left(\Omega_{\varepsilon}\right)
$$

and thus also

$$
a^{i j}(D u) \in H_{0, \alpha}^{(0)}\left(\Omega_{\varepsilon}\right) .
$$

Finally, based on conditions (2.1) and (2.2) the linear theory, i.e. Theorem A.3 yields the estimate in $H_{2, \alpha}^{(-1-\beta)}\left(\Omega_{\varepsilon}\right)$ for some $\beta=\beta\left(D_{\mu} \operatorname{dist}_{E_{0, \varepsilon}}, D_{\mu} \operatorname{dist}_{F_{L_{\varepsilon}}}\right) \in(0,1)$.

In particular, Proposition 3.11 holds in the following situation:

Corollary 3.12. Let $n \geq 2$. Let $\Sigma^{n}$ be given as the graph of a convex $C^{3}$-function which is asymptotic to a cone in the sense that (3.1) holds. Then, an admissible solution of $(\star)_{\varepsilon, \tau}$ with $\varepsilon>0$ sufficiently small satisfies the estimates of Proposition 3.11.

Proof. Under these assumptions we constructed a subsolution $u_{L_{\varepsilon}}$ in Lemma 3.3 which satisfies

$$
u_{L_{\varepsilon}} \geq-\varepsilon^{5 / 4} \text {. }
$$

Furthermore, the gradient estimate on $\Sigma_{\varepsilon}$ is independent of $\varepsilon$ since $\Sigma^{n}$ is convex. Thus, all conditions of Proposition 3.11 are satisfied.

Now we can use the a priori estimates to obtain a unique solution of the approximating problems $(\star)_{\varepsilon, \tau}$. Furthermore, we can use the uniform estimate of $\left|D u^{\varepsilon, \tau}\right|$ to obtain a converging subsequence of solutions as $\varepsilon$ tends to zero.

Proposition 3.13 (Existence for the $(\star)_{\varepsilon, \tau}$ problem). Let $\varepsilon>0$ be sufficiently small. Under the assumptions of Proposition 3.11 there exists a unique solution $u^{\varepsilon, \tau} \in H_{2, \alpha}^{(-1-\beta)}\left(\Omega_{\varepsilon}\right)$ of $(\star)_{\varepsilon, \tau}$ for all $\tau \in\left[0, L_{\varepsilon}\right]$. Furthermore, there exist sequences $\left(\varepsilon_{i}\right)_{i \in \mathbb{N}},\left(L_{\varepsilon_{i}}\right)_{i \in \mathbb{N}},\left(\Omega_{\varepsilon_{i}}\right)_{i \in \mathbb{N}}$ and $\left(u^{\varepsilon_{i}, L_{\varepsilon_{i}}}\right)_{i \in \mathbb{N}}$ such that for $\varepsilon_{i} \rightarrow 0$ we have $L_{\varepsilon_{i}} \rightarrow \infty$,

$$
F_{L_{\varepsilon_{i}}} \backslash E_{0, \varepsilon_{i}} \rightarrow \Omega \backslash E_{0} \quad \text { and } \quad u^{\varepsilon_{i}, L_{\varepsilon_{i}}} \rightarrow u \in C_{\mathrm{loc}}^{0,1}\left(\Omega \backslash E_{0}\right)
$$

locally uniformly.

Proof. Let $\varepsilon>0$ be sufficiently small. We proceed in two steps. First we prove the existence of solutions for $\tau=0$. In the second step we consider $\tau \in\left[0, L_{\varepsilon}\right]$. The equation $Q^{\varepsilon}(u)=0$ is equivalent to $F(u / \varepsilon)=\varepsilon$ with

$$
F(u):=\frac{1}{\sqrt{1+|D u|^{2}}} \operatorname{div}\left(\frac{D u}{\sqrt{1+|D u|^{2}}}\right) .
$$


Therefore, the function $u$ is a solution of $(\star)_{\varepsilon, 0}$ if and only if $\hat{u}:=u / \varepsilon$ solves

$\widehat{(\star)_{\varepsilon, 0}}$

$$
\left\{\begin{aligned}
F(\hat{u})=\varepsilon & \text { in } \Omega_{\varepsilon}, \\
D_{\mu} \hat{u}=0 & \text { on } \Sigma_{\varepsilon}, \\
\hat{u}=0 & \text { on } \partial_{\Omega} E_{0, \varepsilon} \cup \partial_{\Omega} F_{L_{\varepsilon}} .
\end{aligned}\right.
$$

We consider $F$ as an operator $F: A \rightarrow B$ where

$$
\begin{aligned}
& A:=\left\{w \in H_{2, \alpha}^{(-1-\beta)}\left(\Omega_{\varepsilon}\right) \mid w=0 \text { on } \partial_{\Omega} E_{0, \varepsilon} \cup \partial_{\Omega} F_{L_{\varepsilon}}, D_{\mu} w=0 \text { on } \Sigma_{\varepsilon}\right\}, \\
& B:=H_{0, \alpha}^{(1-\beta)}\left(\Omega_{\varepsilon}\right) .
\end{aligned}
$$

The weighted Hölder spaces $H_{k, \alpha}^{(b)}(\Omega)$ are discussed in Definition A.1. The value for $\beta$ is the one from Proposition 3.11. The problem $\widehat{(\star)_{0}}$ has the solution $\hat{u}_{0}:=0$. Furthermore, the linearization of $F$ around $\hat{u}_{0}$ is the Laplacian, i.e. $D F_{\hat{u}_{0}}=\Delta$. The linear theory guarantees the global invertibility of $D F$ around $\hat{u}_{0}$. Thus, the Inverse Function Theorem implies the invertibility of $F$ in a neighborhood of $F\left(\hat{u}_{0}\right)=0$ in $B$. This proves the existence of a unique solution of $\widehat{(\star)_{\varepsilon, 0}}$ and thus of $(\star)_{\varepsilon, 0}$ for $\varepsilon>0$ sufficiently small.

To prove the existence of solutions of $(\star)_{\varepsilon, \tau}$ let us define the set

$$
I_{\varepsilon}:=\left\{\tau \in\left[0, L_{\varepsilon}\right] \mid(\star)_{\varepsilon, \tau} \text { has a unique solution in } H_{2, \alpha}^{(-1-\beta)}\left(\Omega_{\varepsilon}\right)\right\} .
$$

We already know that $0 \in I_{\varepsilon}$. If we can show that $I_{\varepsilon}$ is open and closed, we obtain the desired result. To show that $I_{\varepsilon}$ is open we use once more the Inverse Function Theorem. We modify the spaces $A$ and $B$ to allow other boundary values than zero on $\partial_{\Omega} F_{L_{\varepsilon}}$ and define

$$
\begin{aligned}
& A:=\left\{w \in H_{2, \alpha}^{(-1-\beta)}\left(\Omega_{\varepsilon}\right) \mid w=0 \text { on } \partial_{\Omega} E_{0, \varepsilon}, D_{\mu} w=0 \text { on } \Sigma_{\varepsilon}\right\}, \\
& B:=B_{1} \times B_{2}:=H_{0, \alpha}^{(1-\beta)}\left(\Omega_{\varepsilon}\right) \times H_{2, \alpha}^{(-1-\beta)}\left(\partial_{\Omega} F_{L_{\varepsilon}}\right) .
\end{aligned}
$$

We denote the restriction on $\partial_{\Omega} F_{L_{\varepsilon}}$ by

$$
\pi: A \rightarrow B_{2}, \quad w \mapsto \pi(w):=\left.w\right|_{\partial_{\Omega}} F_{L \varepsilon}
$$

and consider the operator

$$
T: A \rightarrow B, \quad w \mapsto T w:=\left(Q^{\varepsilon}(w), \pi(w)\right) .
$$

Its linearization around some $u_{0} \in A$ is given by $D T_{u_{0}} w=\left(D Q_{u_{0}}^{\varepsilon} w, \pi(w)\right)$ with

$$
D Q_{u_{0}}^{\varepsilon} w=a^{i j}\left(D u_{0}\right) D_{i j} w+B^{k}\left(D u_{0}, D^{2} u_{0}\right) D_{k} w .
$$

Let $\tau \in I_{\varepsilon}$. We linearize $T$ around $u_{0}:=u^{\varepsilon, \tau}$. Here it is crucial that $u_{0} \in C^{1, \beta}\left(\overline{\Omega_{\varepsilon}}\right)$ in order to control the ellipticity. The linear theory, Theorem A.3, implies that $D T_{u_{0}}$ is globally invertible. Therefore, $T$ is invertible in a small neighborhood of $T u_{0}=(0, \tau)$. Thus, $I_{\varepsilon}$ is open.

In order to prove that $I_{\varepsilon}$ is closed let us consider a sequence of elements $\tau_{n} \in I_{\varepsilon}$ which is converging to $\tau$. The corresponding sequence of solutions is $u^{\varepsilon, \tau_{n}}$. Using the maximum principle, the a priori estimates of Proposition 3.11 and the Arzelà-Ascoli type result of Lemma A.2 we obtain a subsequence converging to $u^{\varepsilon, \tau} \in A$. Thus, $\tau \in I_{\varepsilon}$ and $I_{\varepsilon}$ is closed.

Using the a priori estimates for $\left|D u^{\varepsilon, L_{\varepsilon}}\right|$ which are independent of $\varepsilon$ we see that $u^{\varepsilon, L_{\varepsilon}}$ is uniformly bounded and uniformly equicontinuous on compact subsets. Thus, by Arzelà-Ascoli there exists a subsequence $u^{\varepsilon_{i}, L_{\varepsilon_{i}}}$ converging to a locally Lipschitz continuous function $u$. 
In particular, Proposition 3.13 holds in the following situation.

Corollary 3.14. Let $n \geq 2$ and let $\varepsilon>0$ be sufficiently small. Let $E_{0}, E_{0, \varepsilon}$ and $(\star)_{\varepsilon, \tau}$ be defined as above. Furthermore, let $\Sigma^{n}$ be given as the graph of a convex $C^{3}$-function which is asymptotic to a cone in the sense that (3.1) holds. Then the conditions of Theorem 3.13 are satisfied.

Proof. By Corollary 3.12 these assumptions assure that the a priori estimates of Proposition 3.11 hold.

\section{Variational characterization of the limit}

In the last section, we obtained a function $u \in C_{\text {loc }}^{0,1}\left(\Omega \backslash E_{0}\right)$ as the limit of solutions $u^{\varepsilon_{i}}$ of the approximating problems $(\star)_{\varepsilon_{i}, L_{\varepsilon_{i}}}$. In this section, we follow the ideas of Huisken and Ilmanen [11] and define weak solutions of $(\star)$. We show that the limit $u$ is the unique weak solution.

Definition 4.1. Let $A \subseteq \Omega$ be relatively open in $\Omega$. For $u \in C_{\text {loc }}^{0,1}(A)$ we consider the functional

$$
J_{u}^{K}: C_{\operatorname{loc}}^{0,1}(A) \rightarrow \mathbb{R}, \quad v \mapsto J_{u}^{K}(v):=\int_{K}(|D v|+v|D u|) \mathrm{d} \lambda
$$

where $\{u \neq v\} \subset K$ and $K \subset A$ is a compact Caccioppoli set. In the following we omit the set $K$ and write $J_{u}$ instead of $J_{u}^{K}$. The function $u \in C_{\text {loc }}^{0,1}(\Omega)$ is called a weak solution of $(\star)$ with initial condition $E_{0} \subset \Omega$ if $E_{0}=\{u<0\}$ and

$$
J_{u}(u) \leq J_{u}(v) \text { for all } v \in C_{\mathrm{loc}}^{0,1}\left(\Omega_{0}\right),\{u \neq v\} \subset \subset \Omega_{0} .
$$

Here $\Omega_{0}:=\Omega \backslash \overline{E_{0}}$. The integration is performed over any set $K$ as above containing $\{u \neq v\}$.

We can give an alternative characterization of weak solutions.

Definition 4.2. Let $A \subseteq \Omega$. For $u \in C_{\mathrm{loc}}^{0,1}(A)$ we consider the functional

$$
J_{u}^{K}: \mathrm{Ca}(A) \rightarrow \mathbb{R}, \quad F \mapsto J_{u}^{K}(F):=\left|\partial_{\Omega}^{*} F \cap K\right|-\int_{F \cap K}|D u| \mathrm{d} \lambda
$$

where $K$ is a compact set such that $\left|\partial_{\Omega}^{*} F \cap \partial K\right|=0$. Here $\mathrm{Ca}(A)$ denotes the set of all Caccioppoli sets in $A$. In the following we omit the set $K$ and write $J_{u}$ instead of $J_{u}^{K}$. Let $\left(E_{t}\right)_{t>0} \subset \Omega$ be a nested family of relatively open Caccioppoli sets in $\Omega$. Let $u$ be defined by $E_{t}=\{u<t\} \subset \Omega$. The family $\left(E_{t}\right)_{t>0}$ is called a weak solution of ( $\star$ ) with initial condition $E_{0} \subset \Omega$ if $u \in C_{\mathrm{loc}}^{0,1}(\Omega)$ and

$$
J_{u}(E) \leq J_{u}(F) \text { for all } F \in \mathrm{Ca}\left(\Omega \backslash E_{0}\right), E \Delta F \subset \subset \Omega \backslash E_{0}
$$

for each $t>0$. The integration is performed over set $K$ as above containing $E \Delta F$.

These two definitions are compatible. 
Lemma 4.3. Let $u \in C_{\mathrm{loc}}^{0,1}(\Omega)$ and let $E_{0} \subset \Omega$ be relatively open in $\Omega$. The following are equivalent:

$(\dagger)(\{u<t\})_{t>0}$ is a weak solution of $(\star)$ with initial condition $E_{0}$.

$(\dagger \dagger) u$ is a weak solution of $(\star)$ with initial condition $E_{0}$.

Proof. See [11, Lemma 1.1 and Lemma 1.2].

The following two results are be needed in the sequel.

Lemma 4.4 (Compactness of weak solutions). Let $\left(A_{i}\right)_{i \in \mathbb{N}}, A \subset \Omega$ be relatively open in $\Omega$. Let $\left(u_{i}\right)_{i \in \mathbb{N}} \subset C_{\mathrm{loc}}^{0,1}\left(A_{i}\right)$ be a sequence of weak solutions of $(\star)$ such that

$$
A_{i} \rightarrow A, \quad u_{i} \rightarrow u \in C_{\mathrm{loc}}^{0,1}(A)
$$

locally uniformly for $i \rightarrow \infty$. If for each compact set $K \subset A$ and $i$ large enough

$$
\operatorname{ess} \sup _{K}\left|D u_{i}\right| \leq C(K)
$$

then $u$ is a weak solution of $(\star)$ in $A$.

Proof. See [11, Theorem 2.1].

Lemma 4.5 (Uniqueness of weak solutions). Let $A \subset \Omega$ be relatively open in $\Omega$. For a given $E_{0} \subset \Omega$, there exists at most one solution $\left(E_{t}\right)_{t>0} \subset \Omega$ of $(\dagger)$ such that each $E_{t}$ is precompact.

Proof. See [11, Theorem 2.2].

Note that the assumption of $E_{t}$ being precompact cannot be dropped: If $u \in C_{\mathrm{loc}}^{0,1}(\Omega)$ satisfies (††) and $t>0$, then $\min (u(\cdot), t)$ also satisfies $(\dagger \dagger)$. Next, we show that the definition of weak solutions is compatible with classical solutions.

Lemma 4.6 (Classical $\Rightarrow$ weak). Let $\left(N_{t}\right)_{c \leq t \leq d} \subset \Omega$ be a family of compact surfaces of positive mean curvature that solve (IMCF) classically. Let $u=t$ on $N_{t}, u<c$ in the region bounded by $N_{c}$, and $E_{t}:=\{u<t\} \subset \Omega$. Then for $c<t<d$, $E_{t}$ minimizes $J_{u}$ in $E_{d} \backslash \overline{E_{c}}$.

Proof. Let $t \in(c, d)$. We have to show that $E_{t}:=\{u<t\}$ minimizes $J_{u}$ in $E_{d} \backslash \overline{E_{c}}$, i.e.

$$
\left|\partial_{\Omega}^{*} E_{t} \cap K\right|-\int_{E_{t} \cap K}|D u| \mathrm{d} \lambda \leq\left|\partial_{\Omega}^{*} F \cap K\right|-\int_{F \cap K}|D u| \mathrm{d} \lambda
$$

for all $F$ having locally finite perimeter and satisfying $E_{t} \Delta F \subset \subset E_{d} \backslash \overline{E_{c}}$. We choose $r, s \in \mathbb{R}$ such that $c<r<t<s<d$ and use $K:=\overline{E_{s} \backslash E_{r}}$. Then the above inequality reads

$$
\left|\partial_{\Omega}^{*} E_{t}\right|-\int_{E_{t} \backslash E_{r}}|D u| \mathrm{d} \lambda \leq\left|\partial_{\Omega}^{*} F\right|-\int_{F \backslash E_{r}}|D u| \mathrm{d} \lambda .
$$

Let us consider the vector field $X:=D u /|D u|$ which is $C^{1}$ away from $\partial_{\Omega} E_{c} \cap \partial_{\Sigma} E_{c}$ and $\partial_{\Omega} E_{d} \cap \partial_{\Sigma} E_{d}$. The Divergence Theorem and the fact that $u$ is a solution of ( $\star$ ) yield

$$
\int_{\partial A} v_{\partial A} \cdot X \mathrm{~d} s=\int_{A} \operatorname{div}(X) \mathrm{d} \lambda=\int_{A}|D u| \mathrm{d} \lambda
$$


Furthermore, for any set $A \subset \Omega$ we have

$$
\int_{\partial_{\Sigma} A} v_{\partial_{\Sigma} A} \cdot X \mathrm{~d} s=\int_{\partial_{\Sigma} A} \mu \cdot \frac{D u}{|D u|} \mathrm{d} s=0
$$

These two equalities help us to calculate

$$
\begin{aligned}
\left|\partial_{\Omega}^{*} E_{t}\right|-\int_{E_{t} \backslash E_{r}}|D u| \mathrm{d} \lambda & =\int_{\partial_{\Omega}^{*} E_{t}} v_{\partial_{\Omega}^{*} E_{t}} \cdot X \mathrm{~d} s-\int_{E_{t} \backslash E_{r}}|D u| \mathrm{d} \lambda \\
& \leq \int_{\partial_{\Omega}^{*} F} v_{\partial_{\Omega}^{*} F} \cdot X \mathrm{~d} s-\int_{F \backslash E_{r}}|D u| \mathrm{d} \lambda \\
& \leq\left|\partial_{\Omega}^{*} F\right|-\int_{F \backslash E_{r}}|D u| \mathrm{d} \lambda .
\end{aligned}
$$

This shows that $E_{t}$ minimizes $J_{u}$ in $E_{d} \backslash \overline{E_{c}}$.

Now we are able to prove that the limit $u$ which was obtained in the previous section is a weak solution of $(\star)$ in $\Omega_{0}$.

Proposition 4.7 (Criterion for existence). Let $\left(u_{i}\right)_{i \in \mathbb{N}} \subset H_{2, \alpha}^{(-1-\beta)}\left(\Omega_{\varepsilon_{i}}\right)$ be a sequence of solutions of $(\star)_{\varepsilon_{i}}, L_{\varepsilon_{i}}$ with

$$
F_{L_{\varepsilon_{i}}} \backslash E_{0, \varepsilon_{i}} \rightarrow \Omega \backslash E_{0}, \quad u_{i} \rightarrow u \in C_{\mathrm{loc}}^{0,1}\left(\Omega \backslash E_{0}\right)
$$

locally uniformly for $i \rightarrow \infty$. If for each compact set $K \subset \Omega \backslash E_{0}$ and $i$ large enough

$$
\sup _{K}\left|D u_{i}\right| \leq C(K)
$$

then $u$ is a weak solution of $(\star)$ in $\Omega_{0}:=\Omega \backslash \overline{E_{0}}$ with initial condition $E_{0}$.

Proof. Let us define

$$
\begin{aligned}
U_{i}: \overline{\Omega_{\varepsilon_{i}}} \times \mathbb{R} & \rightarrow \mathbb{R}, \quad(x, z) \mapsto U_{i}(x, z):=u_{i}(x)-\varepsilon_{i} z, \\
U:\left(\Omega \backslash E_{0}\right) & \times \mathbb{R} \rightarrow \mathbb{R}, \quad(x, z) \mapsto U(x, z):=u(x) .
\end{aligned}
$$

Then $U_{i} \rightarrow U$ locally uniformly in $\left(\Omega \backslash E_{0}\right) \times \mathbb{R}$. For fixed $i \in \mathbb{N}$ we consider the sets

$$
M_{t}^{i}:=\left\{(x, z) \in \overline{\Omega_{\varepsilon_{i}}} \times \mathbb{R} \mid U_{i}(x, z)=t\right\}=\operatorname{graph}\left(\frac{u_{i}}{\varepsilon_{i}}-\frac{t}{\varepsilon_{i}}\right) .
$$

Note that these graphs are classical solutions of inverse mean curvature flow one dimension higher. This can be seen by computing

$$
\operatorname{div}_{\mathbb{R}^{n+2}}\left(\frac{D U_{i}}{\left|D U_{i}\right|}\right)=\left|D U_{i}\right|
$$

which is equivalent to (IMCF) since $\left|D U_{i}\right|=H>0$ (see Remark 2.2). The Neumann condition is satisfied as well since the normal to $\Sigma^{n} \times \mathbb{R}$ is given by $\widehat{\mu}=(\mu, 0)$ and

$$
D_{\widehat{\mu}} U_{i}=D_{\mu} u_{i}=0 \quad \text { on } \partial_{\Sigma} \Omega_{\varepsilon_{i}} \times \mathbb{R} .
$$

Therefore, Lemma 4.6 implies that $U_{i}$ is a weak solution in $\left(F_{L_{\varepsilon_{i}}} \backslash \overline{E_{0, \varepsilon_{i}}}\right) \times \mathbb{R}$ and thus the 
compactness result, Lemma 4.4 implies that $U$ is a weak solution in $\left(\Omega \backslash \overline{E_{0}}\right) \times \mathbb{R}$. To deduce that $u$ is a weak solution in $\Omega_{0}:=\Omega \backslash \overline{E_{0}}$ we use the cutoff functions

$$
\Phi_{s}: \mathbb{R} \rightarrow \mathbb{R}, \quad z \mapsto \Phi_{s}(z):= \begin{cases}1 & \text { for } z \in[0, s] \\ \Phi(s) & \text { for } z \in[-1,0], \\ \Phi(s-z) & \text { for } z \in[s, s+1], \\ 0 & \text { for } z \in \mathbb{R} \backslash[-1, s+1],\end{cases}
$$

where $\Phi$ is chosen such that $\Phi_{s} \in C^{1}(\mathbb{R})$ with $\Phi_{s}(z) \in[0,1]$ and $\left|\Phi_{s}^{\prime}(z)\right| \leq 2$ for all $z \in \mathbb{R}$. As a competitor to $U(x, z)=u(x)$ we use

$$
V: \Omega_{0} \times \mathbb{R}, \quad(x, z) \mapsto V(x, z):=\Phi_{S}(z) v(x)+\left(1-\Phi_{S}(z)\right) u(x)
$$

where $v \in L_{\text {loc }}^{0,1}\left(\Omega_{0}\right)$ with $\{u \neq v\} \subset K$ and $K$ a compact subset of $\Omega_{0}$. We compute that

$$
\left|D_{x, z} V\right| \leq \Phi_{s}\left|D_{x} v\right|+\left(1-\Phi_{s}\right)\left|D_{x} u\right|+\left|\Phi_{s}^{\prime}\right||v-u| .
$$

Since $\{U \neq V\} \subset K \times[-1, s+1] \subset \subset \Omega_{0} \times \mathbb{R}$, we have

$$
J_{U}(U) \leq J_{U}(V),
$$

i.e.

$$
\begin{aligned}
& \int_{K \times[-1, s+1]} \Phi_{s}\left(\left|D_{x} u\right|+u\left|D_{x} u\right|\right) \mathrm{d} \lambda(x, z) \\
& \quad \leq \int_{K \times[-1, s+1]}\left(\Phi_{s}\left(\left|D_{x} v\right|+v\left|D_{x} u\right|\right)+\left|\Phi_{s}^{\prime}\right||v-u|\right) \mathrm{d} \lambda(x, z) .
\end{aligned}
$$

This implies

$$
s J_{u}(u) \leq(s+2) J_{u}(v)+4 \int_{K}|v-u| \mathrm{d} \lambda(x) .
$$

Dividing by $s$ and passing $s \rightarrow \infty$ proves that $J_{u}(u) \leq J_{u}(v)$. Finally, we extend $u$ negatively to $E_{0}$ in order to satisfy $E_{0}=\{u<0\}$.

Finally, we can state and prove our main existence and uniqueness result for weak solutions of inverse mean curvature flow for hypersurfaces with boundary:

Theorem 4.8 (Existence and uniqueness of weak solutions). Let $\Sigma^{n}, E_{0}, E_{0, \varepsilon}$ and $(\star)_{\varepsilon, \tau}$ be defined as above. Assume that an admissible subsolution $u_{L_{\varepsilon}}$ of problem $(\star)_{\varepsilon, L_{\varepsilon}}$ exists such that $F_{L_{\varepsilon}}=\left\{u_{L_{\varepsilon}}<L_{\varepsilon}\right\}$ satisfies condition (2.2). Let $u$ be an admissible solution of problem $(\star)_{\varepsilon, \tau}$ such that $u \geq-\varepsilon^{1+\gamma}$ for some $\gamma \in(0,1)$ and that $|D u|_{\Sigma_{\varepsilon}} \leq c_{1}$ independently of $\varepsilon$. Then there exists a weak solution $u \in C_{\mathrm{loc}}^{0,1}(\Omega)$ of problem $(\star)$ with initial condition $E_{0}$ such that for all $t>0$ the sets $E_{t}:=\{u<t\}$ are the unique precompact minimizers of $J_{u}$ in $\Omega \backslash E_{0}$.

Proof. Proposition 3.13 provides a sequence of solutions $\left(u_{i}\right)_{i \in \mathbb{N}}$ of $(\star)_{\varepsilon_{i}, L_{\varepsilon_{i}}}$ which converges locally uniformly to a function $u \in C_{\mathrm{loc}}^{0,1}\left(\Omega \backslash E_{0}\right)$. Then Lemma 4.7 implies that $u$ is a weak solution of $(\star)$ in $\Omega_{0}:=\Omega \backslash \overline{E_{0}}$ with initial condition $E_{0}$. Finally, Lemma 4.5 shows that $u$ is the unique weak solution as long as $E_{t}$ remains precompact. 
In particular, Theorem 4.8 holds in the following situation.

Corollary 4.9. Let $n \geq 2$ and let $\varepsilon>0$ be sufficiently small. Let $E_{0}, E_{0, \varepsilon}$ and $(\star)_{\varepsilon, \tau}$ be defined as above. Furthermore, let $\Sigma^{n}$ be given as the graph of a convex $C^{3}$-function which is asymptotic to a cone in the sense that (3.1) holds. Then the conditions of Theorem 4.8 are satisfied.

Proof. By Corollary 3.14 the assumptions assure that all conditions in Proposition 3.13 are satisfied.

\section{Properties of weak solutions}

For minimizers of the functional $J_{u}$ we obtain the following regularity.

Lemma 5.1. Let $u \in C_{\mathrm{loc}}^{0,1}(A)$. Let $E \subset \Omega$ be a minimizer of the functional $J_{u}$. Then $\partial_{\Omega}^{*} E$ is a subset of a $C^{1,1 / 2}$-hypersurface and

$$
\mathscr{H}^{k}\left(\partial_{\Omega} E \backslash \partial_{\Omega}^{*} E\right)=0 \quad \text { for all } k>n-8
$$

where $\partial_{\Omega}^{*} E$ is the reduced boundary of $E$ in $\Omega$.

Proof. Since $u \in C_{\text {loc }}^{0,1}(A)$, we see that minimizers of $J_{u}$ are almost minimal in the sense that for balls of radius $R$ we have

$$
\left|\partial_{\Omega}^{*} E \cap B_{R}\right| \leq\left|\partial_{\Omega}^{*} F \cap B_{R}\right|+C\left(\|D u\|_{\infty}, n\right) R^{n+1}
$$

for $E \Delta F \subset \subset B_{R}$. Thus [32, Theorem 1] yields the result. See also [24].

For classical solutions of (IMCF) we have $H=|D u|$. Now we want to show that this equality still holds in a weak sense for minimizers of $J_{u}$. Recall, that for $C^{2}$-submanifolds $M^{n}$ of $\mathbb{R}^{n+1}$ with $(n-1)$-dimensional $C^{1}$-boundary $\partial M^{n}$ and $C^{1}$-vector fields $X$ the following equality holds:

$$
\int_{M^{n}}\left(\operatorname{div}_{M^{n}} X-H v \cdot X\right) \mathrm{d} \mu=-\int_{\partial M^{n}} X \cdot \eta \mathrm{d} s
$$

where $\eta$ is the inward pointing unit co-normal of $\partial M^{n}$ (see [29]). Note that if $M^{n}$ and $\Sigma^{n}$ met orthogonally, the right-hand side would vanish for variations $X$ which are tangential along $\Sigma^{n}$.

Definition 5.2. We say that the hypersurface $M^{n}$ possesses a weak mean curvature in $L^{p}$ if there exists a vector-valued function $H \in L_{\mathrm{loc}}^{p}\left(M^{n}, \mathbb{R}^{n+1}\right)$ such that

$$
\int_{M^{n}}\left(\operatorname{div}_{M^{n}} X-H \cdot X\right) \mathrm{d} \mu=0
$$

for all $X \in C_{c}^{\infty}\left(T M^{n}\right)$ with spt $X \cap \partial M^{n}=\emptyset$. Furthermore, we say that $M^{n}$ is weakly orthogonal to $\Sigma^{n}$ if condition (5.2) holds for all $X \in C_{c}^{\infty}\left(T M^{n}\right)$ which are tangential along $\Sigma^{n}$, i.e. $X(x) \in T_{x} \Sigma^{n}$ for $x \in \Sigma^{n}$. 
Lemma 5.3 (Weak mean curvature). Let $a, b \in \mathbb{R}_{+}$with $a<b$ and let $E_{t}:=\{u<t\}$ minimize $J_{u}$ in $A:=E_{b} \backslash E_{a}$ where $u \in C_{\mathrm{loc}}^{0,1}(A)$. Then up to a set of dimension less than or equal to $n-8, M_{t}^{n}:=\partial_{\Omega} E_{t}$ is a $C^{1,1 / 2}$-hypersurface which possesses a weak mean curvature in $L^{\infty}$ given by

$$
H(x)=|D u(x)| v(x) \quad \text { where } v(x):=\frac{D u(x)}{|D u(x)|}
$$

for almost every $t \in(a, b)$ and almost every $x \in M_{t}^{n}$. Furthermore, for those values of $t, M_{t}^{n}$ is orthogonal to $\Sigma^{n}$ in the classical sense in any neighborhood of points $x \in \partial_{\Omega}^{*} E_{t} \cap \Sigma^{n}$.

Proof. Let $U \subset \mathbb{R}^{n+1}$ be open such that $U \cap A \neq \emptyset$, and let $K \subset U$ be compact such that $K \cap M_{t}^{n} \neq 0$. We consider a family of diffeomorphisms

$$
\Phi:(-1,1) \times U \rightarrow U, \quad(x, s) \mapsto \Phi(s, x)=: \Phi_{s}(x)
$$

satisfying

$$
\Phi_{0}=\mathrm{id},\left.\quad \Phi_{S}\right|_{U \backslash K}=\left.\mathrm{id}\right|_{U \backslash K},\left.\quad \frac{\partial \Phi(s, x)}{\partial s}\right|_{s=0}=X\left(\Phi_{0}(x)\right)=X(x)
$$

where $X$ is a smooth vector field with support in $K$ and tangential to $\Sigma^{n}$ if $K \cap \Sigma^{n} \neq \emptyset$. Note that

$$
\left.\frac{\partial \Phi_{s}^{-1}(y)}{\partial s}\right|_{s=0}=-X\left(\Phi_{0}^{-1}(y)\right)=-X(y) .
$$

By Lemma 4.3 the function $u$ minimizes $J_{u}$ in $E_{b} \backslash \overline{E_{a}}$. Therefore, the first variation of $J_{u}$ vanishes. We use the area and co-area formula to compute

$$
\begin{aligned}
0 & =\left.\frac{\mathrm{d}}{\mathrm{d} s}\right|_{s=0} J_{u}\left(u \circ \Phi_{s}^{-1}\right) \\
& =\left.\frac{\mathrm{d}}{\mathrm{d} s}\right|_{s=0}\left(\int_{U}|D u(x)| \cdot\left|\operatorname{det} D \Phi_{s}(x)\right| \mathrm{d} \lambda(x)+\int_{a}^{b} \int_{M_{t}^{n} \cap \Phi_{s}(U)}\left(u \circ \Phi_{s}^{-1}\right)(y) \mathrm{d} \mathscr{H}^{n}(y) \mathrm{d} t\right) \\
& =\int_{a}^{b} \int_{M_{t}^{n} \cap U}\left(\operatorname{div}_{M_{t}^{n}} X(x)-D u(x) \cdot X(x)\right) \mathrm{d} \mathscr{H}^{n}(x) \mathrm{d} t .
\end{aligned}
$$

The Lebesgue Differentiation Theorem implies that the inner integral vanishes for almost every $t \in(a, b)$. Thus, a comparison with (5.2) yields the result. The fact that we obtained (5.2) for all vector fields which are tangential to $\Sigma^{n}$ shows that $M_{t}^{n}$ is weakly orthogonal to $\Sigma^{n}$. Combining the fact that $E_{t}$ is almost minimal, i.e. (5.1) with the existence of a weak mean curvature in $L^{\infty}$ one can argue as in [8] or [7] and apply the results of [9] to prove the regularity result of Lemma 5.1 up to the boundary of $M_{t}^{n}$. This implies that $M_{t}^{n}$ meets $\Sigma^{n}$ orthogonally in the classical sense in any neighborhood of points in $\partial_{\Omega}^{*} E_{t} \cap \Sigma^{n}$.

Now we come to a geometric characterization of the jumps of the hypersurfaces which occur under the weak flow.

Definition 5.4. Let $A \subseteq \Omega$ be relatively open in $\Omega$. The set $E \subset \Omega$ is called a minimizing hull in $A$ if for all sets $F \subset \Omega$ and all compact sets $K \subset A$ containing $F \backslash E$ we have

$$
\left|\partial_{\Omega}^{*} E \cap K\right| \leq\left|\partial_{\Omega}^{*} F \cap K\right| \text { for } F \supseteq E .
$$


Furthermore, $E$ is called a strictly minimizing hull in $A$ if $E$ is a minimizing hull in $A$ and in addition

$$
\left|\partial_{\Omega}^{*} E \cap K\right|=\left|\partial_{\Omega}^{*} F \cap K\right| \Longrightarrow E \cap A=F \cap A .
$$

Let $E \subseteq \Omega$ be some measurable set and let $A \subseteq \Omega$ be relatively open in $\Omega$. We consider the family $\left(E_{\iota}\right)_{\iota \in J}$ of the Lebesgue points of strictly minimizing hulls in $A$ which contain $E$. Using this family we define the strictly minimizing hull of $E$ in $A$ as

$$
E_{A}^{\prime}:=\bigcap_{\iota \in J} E_{\iota}
$$

Note that up to a set of measure zero $E_{A}^{\prime}$ may be realized by a countable intersection and therefore $E_{A}^{\prime}$ is a strictly minimizing hull and open (compare with [1, Definition 2.1]).

The following lemma provides a description of the jumping time.

Lemma 5.5 (Minimizing hull property). Let $u \in C_{\text {loc }}^{0,1}(\Omega)$ satisfy (††). Then:

(1) For $t>0, E_{t}:=\{u<t\}$ is a minimizing hull in $\Omega$.

(2) For $t \geq 0, E_{t}^{+}:=\operatorname{int}\{u \leq t\}$ is a strictly minimizing hull in $\Omega$.

(3) For $t \geq 0, E_{t}^{\prime}=E_{t}^{+}$, provided that $E_{t}^{+}$is precompact.

(4) For $t>0,\left|\partial_{\Omega}^{*} E_{t}\right|=\left|\partial_{\Omega}^{*} E_{t}^{+}\right|$provided that $E_{t}^{+}$is precompact.

Note that 4) holds for $t=0$ if, and only if, $E_{0}$ is a minimizing hull.

Proof. See [11, Minimizing Hull Property 1.4].

As for the classical flow the rescaled surface area is constant:

Lemma 5.6 (Exponential growth). Let $\left(E_{t}\right)_{t>0}$ solve $(\dagger)$ with initial condition $E_{0}$. As long as $E_{t}$ remains precompact, we have

$$
\left|\partial_{\Omega}^{*} E_{t}\right|=c e^{t}, \quad c \in \mathbb{R}, t>0 .
$$

If $E_{0}$ is a minimizing hull, then $c=\left|\partial_{\Omega}^{*} E_{0}\right|$.

Proof. See [11, Lemma 1.6].

\section{Outlook: Monotonicity of the Hawking mass}

The evolution of closed hypersurfaces under inverse mean curvature flow (IMCF) was put forward by Geroch [5] and Jang and Wald [13] as an approach to the proof of the Positive Mass Theorem. They observed the monotonicity of the Hawking mass

$$
m_{\text {Haw }}\left(M^{2}\right):=\frac{\left|M^{2}\right|^{1 / 2}}{(16 \pi)^{3 / 2}}\left(16 \pi-\int_{M^{2}} H^{2} \mathrm{~d} \mu\right)
$$

under IMCF and showed that if IMCF remained smooth, this monotonicity could be used to prove the Riemannian Penrose inequality and thus the Positive Mass Theorem. Unfortunately, 
IMCF does not remain smooth in general. However, the Positive Mass Theorem was proved by Schoen and Yau [26] using a different approach. The big achievement of Huisken and Ilmanen [11] was to define weak solutions of IMCF which still keep $m_{\text {Haw }}$ monotone. This enabled them to prove the Riemannian Penrose inequality. See also Bray [2] for a different approach.

In the following we introduce an analog quantity of $m_{\text {Haw }}$. We prove its monotonicity under classical IMCF for hypersurfaces with boundary. The justification of the monotonicity in the weak setting will be carried out in a forthcoming paper.

Proposition 6.1. Let $\Sigma^{2}, M_{0}^{2} \subset \mathbb{R}^{3}$ be orientable $C^{2, \alpha}$-surfaces. Suppose $\partial M_{0}^{2} \subset \Sigma^{2}$ such that $M_{0}^{2}$ touches $\Sigma^{2}$ orthogonally. Let $\left(M_{t}^{2}\right)_{t \geq 0} \subset \mathbb{R}^{3}$ be a classical solution of (IMCF) such that each $M_{t}^{2}$ is connected. If $\Sigma^{2}$ is mean-convex, i.e. ${ }^{\Sigma^{2}} H \geq 0$, then the quantity

$$
\left|M_{t}^{2}\right|^{1 / 2}\left(8 \pi-\int_{M_{t}^{2}} H^{2} \mathrm{~d} \mu_{t}\right)
$$

is monotone increasing in $t$.

Proof. Note that

$$
\frac{\partial H}{\partial t}=\frac{\Delta H}{H^{2}}-\frac{|A|^{2}}{H}-\frac{2|\nabla H|^{2}}{H^{3}} \text { and } \nabla_{\mu} H=-H^{\Sigma^{2}} h(\nu, v) .
$$

The evolution equation can be found in [11] and the formula for $\nabla_{\mu} H$ follows from differentiating $0=\mu \cdot v$ in time:

$$
0=\frac{\mathrm{d}}{\mathrm{d} t}\langle\nu, \mu\rangle=\left\langle\frac{\mathrm{d} v}{\mathrm{~d} t}, \mu\right\rangle+\left\langle\nu, \frac{\mathrm{d} \mu}{\mathrm{d} t}\right\rangle=\left\langle\frac{\nabla H}{H^{2}}, \mu\right\rangle+\left\langle v, \frac{\nabla_{\nu} \mu}{H}\right\rangle .
$$

Furthermore, we use the Gauss equation $2 K=H^{2}-|A|^{2}$ and the Gauss-Bonnet formula

$$
\int_{M^{2}} K \mathrm{~d} \mu=2 \pi-\int_{\partial M^{2}} k_{g} \mathrm{~d} s=2 \pi-\int_{\partial M^{2}} \Sigma^{2} h(\tau, \tau) \mathrm{d} s .
$$

Here $k_{g}$ is the geodesic curvature of the boundary curve. It can be expressed as the second fundamental form of $\Sigma^{2}$ in direction $\tau \in T M^{2} \cap T \Sigma^{2}$ where $|\tau|=1$. Putting everything together we obtain

$$
\begin{aligned}
\frac{\mathrm{d}}{\mathrm{d} t} \int_{M_{t}^{2}} H^{2} \mathrm{~d} \mu_{t} & =\int_{M_{t}^{2}}\left(H^{2}+2 H\left[\frac{\Delta H}{H^{2}}-\frac{2|\nabla H|^{2}}{H^{3}}-\frac{|A|^{2}}{H}\right]\right) \mathrm{d} \mu_{t} \\
& =\int_{M_{t}^{2}}\left(H^{2}-2|A|^{2}-2 \frac{|\nabla H|^{2}}{H^{2}}\right) \mathrm{d} \mu_{t}+2 \int_{\partial M_{t}^{2}} H^{-1} \nabla_{\mu} H \mathrm{~d} s_{t} \\
& \leq \int_{M_{t}^{2}}\left(2 K-\frac{H^{2}}{2}\right) \mathrm{d} \mu_{t}-2 \int_{\partial M_{t}^{2}} \Sigma^{2} h(v, v) \mathrm{d} s_{t} \\
& \leq \frac{1}{2}\left(8 \pi-\int_{M_{t}^{2}} H^{2} \mathrm{~d} \mu_{t}\right)-2 \int_{\partial M_{t}^{2}} \Sigma^{2} H \mathrm{~d} s_{t} .
\end{aligned}
$$

Using the exponential growth of $\left|M_{t}^{2}\right|$ proven in Lemma 5.6 and the fact that $\Sigma^{2}$ is meanconvex yields the desired monotonicity. 
In case that $\Sigma^{2}$ is convex we obtain

$$
\frac{\mathrm{d}}{\mathrm{d} t} \int_{M_{t}^{2}} H^{2} \mathrm{~d} \mu_{t} \leq 0
$$

This does not require the Gauss-Bonnet formula and is therefore valid in all dimensions. Note that Proposition 6.1 still holds if we replace $\mathbb{R}^{3}$ by a Riemannian 3-manifold with positive scalar curvature.

\section{A. Linear mixed Dirichlet-Neumann problems}

Definition A.1. Let $\Omega \subset \mathbb{R}^{n}$ be a bounded Lipschitz domain. We denote by $\Sigma$ a relatively open part of $\partial \Omega$ and write $\sigma=\partial \Omega \backslash \bar{\Sigma}$. Let $\mu$ be the outward pointing unit normal to $\Omega$ on $\Sigma$. We consider the following mixed Dirichlet-Neumann boundary value problem:

$$
\left\{\begin{aligned}
L u:=a^{i j} D_{i j} u+b^{k} D_{k} u & =f & & \text { in } \Omega, \\
\mu^{k} D_{k} u & =0 & & \text { on } \Sigma, \\
u & =v & & \text { on } \bar{\sigma},
\end{aligned}\right.
$$

where $L$ is assumed to be uniformly elliptic and $\Sigma, \sigma$ are supposed to be subsets of $C^{2, \alpha}$-hypersurfaces. Since the domain might have corners, we introduce weighted Hölder spaces to allow for less regular solutions. For $\delta>0$ sufficiently small we define

$$
\Omega_{\delta}:=\{x \in \Omega \mid \operatorname{dist}(x, \partial \Omega \backslash \Sigma)>\delta\} .
$$

Using the classical Hölder norms $\|\cdot\|_{k, \alpha ; \bar{\Omega}}$ as they appear in [17] we define

$$
\|u\|_{k, \alpha ; \Omega}^{(b)}:=\sup _{\delta>0} \delta^{b+k+\alpha}\|u\|_{k, \alpha ; \overline{\Omega_{\delta}}}, \quad H_{k, \alpha}^{(b)}(\Omega):=\left\{u \mid\|u\|_{k, \alpha ; \Omega}^{(b)}<\infty\right\}
$$

for $k \in \mathbb{N}, \alpha \in(0,1)$ and $b>-k-\alpha$.

These norms have the following useful properties.

Lemma A.2. Let $k_{1}, k_{2}, k, l \in \mathbb{N}$ and $\alpha, \beta \in(0,1)$. If $k+\alpha \geq l+\beta$, then

$$
H_{k, \alpha}^{(-l-\beta)}(\Omega) \subset C^{l, \beta}(\bar{\Omega}) \cap C^{k, \alpha}(\Omega) .
$$

Let $k_{1}+\alpha \geq b>0$. If $\left(u_{n}\right)_{n \in \mathbb{N}} \subset H_{k_{1}, \alpha}^{(-b)}(\Omega)$ is bounded, then there is a subsequence $\left(u_{n_{k}}\right)_{k \in \mathbb{N}}$ such that

$$
u_{n_{k}} \stackrel{H_{k_{2}, \beta}^{\left(-b^{\prime}\right)}(\Omega)}{\longrightarrow} u \quad(k \rightarrow \infty)
$$

for $0<b^{\prime}<b, 0<k_{2}+\beta<k_{1}+\alpha$ and $k_{2}+\beta \geq b^{\prime}$.

Proof. See [18, Section 1] and the introduction of [19].

Now we can state the existence and regularity result for mixed elliptic boundary value problems which is due to Lieberman [20,21]. 
Theorem A.3. Let $\Sigma, \sigma$ be subsets of $C^{2, \alpha}$-hypersurfaces. Let $\Omega \subset \mathbb{R}^{n}$ be a bounded Lipschitz domain with boundary $\partial \Omega=\bar{\sigma} \cup \bar{\Sigma}$ where $\sigma$ and $\Sigma$ are relatively open in $\partial \Omega$. Assume that $a^{i j}$ is uniformly continuous in $\Omega$ and that $L$ is uniformly elliptic. Furthermore, assume that for all $x \in V:=\bar{\sigma} \cap \bar{\Sigma}$ the boundary parts $\sigma$ and $\Sigma$ enclose the domain at an angle $0<\theta(x) \leq \theta_{\max }<\frac{\pi}{2}$. Then there exists some $\beta\left(\theta_{\max }\right) \in(0,1)$ such that if

$$
a^{i j} \in H_{0, \alpha}^{(0)}(\Omega), \quad b^{i} \in H_{0, \alpha}^{(1-\beta)}(\Omega), \quad f \in H_{0, \alpha}^{(1-\beta)}(\Omega), \quad v \in C^{1, \beta}(\bar{\Omega}),
$$

then there exists a unique solution $u \in C^{0}(\bar{\Omega}) \cap C^{2}(\Omega \cup \Sigma)$ of (A.1). Furthermore, each such solution of (A.1) satisfies the estimate

$$
\|u\|_{2, \alpha ; \Omega}^{(-1-\beta)} \leq C\left(\|f\|_{0, \alpha ; \Omega}^{(1-\beta)}+\|v\|_{1, \beta ; \bar{\Omega}}\right) .
$$

Proof. The existence and uniqueness result can be found in [20, Theorem 2]. The regularity result is a variant of [21, Theorem 4]. It relies on a modification of the height estimate [21, Lemma 3.3]. This modification is necessary in order to match with the definition of the weighted norm which is used in [20].

\section{References}

[1] R. Bassanezi and I. Tamanini, Subsolutions to the least area problem and the minimal hull of a bounded set in $\mathbb{R}^{n}$, Ann. Univ. Ferrara Sez. VII Sci. Mat. 30 (1984), 27-40.

[2] H. L. Bray, Proof of the Riemannian Penrose inequality using the positive mass theorem, J. Differential Geom. 59 (2001), no. 2, 177-267.

[3] J.A. Buckland, Mean curvature flow with free boundary on smooth hypersurfaces, Ph.D. thesis, Monash University, 2003.

[4] C. Gerhardt, Flow of nonconvex hypersurfaces into spheres, J. Differential Geom. 32 (1990), no. 1, $299-314$.

[5] R. Geroch, Energy extraction, Ann. New York Acad. Sci. 224 (1973), 108-117.

[6] D. Gilbarg and N.S. Trudinger, Elliptic partial differential equations of second order, Classics Math., Springer, New York 2001.

[7] M. Grüter, Boundary regularity for solutions of a partitioning problem, Arch. Ration. Mech. Anal. 97 (1987), no. 3, 261-270.

[8] M. Grüter, Optimal regularity for codimension one minimal surfaces with a free boundary, Manuscripta Math. 58 (1987), 295-343.

[9] M. Grüter and J. Jost, Allard type regularity results for varifolds with free boundaries, Ann. Sc. Norm. Super. Pisa Cl. Sci. (4) 13 (1986), no. 1, 129-169.

[10] B. Hein, A homotopy approach to solving the inverse mean curvature flow, Calc. Var. Partial Differential Equations 28 (2007), no. 2, 249-273.

[11] G. Huisken and T. Ilmanen, The inverse mean curvature flow and the Riemannian Penrose inequality, J. Differential Geom. 59 (2001), 353-437.

[12] G. Huisken and T. Ilmanen, Higher regularity of the inverse mean curvature flow, J. Differential Geom. 80 (2008), 433-451.

[13] P. S. Jang and R. M. Wald, The positive energy conjecture and the cosmic censor hypothesis, J. Math. Phys. 18 (1977), 41-44.

[14] A. N. Koeller, On the singularity sets of minimal surfaces and a mean curvature flow, Ph.D. thesis, FU Berlin, 2007.

[15] A. N. Koeller, Regularity of mean curvature flows with Neumann free boundary conditions, Calc. Var. Partial Differential Equations 43 (2012), no. 1-2, 265-309.

[16] B. Kotschwar and $L$. Ni, Local gradient estimates of $p$-harmonic functions, $1 / H$ flow, and an entropy formula, Ann. Sci. Éc. Norm. Supér. (4) 42 (2009), 1-36.

[17] O.A. Ladyženskaja and N.N. Ural'ceva, Linear and quasilinear elliptic equations, Math. Sci. Engin. 46, Academic Press, New York 1968.

[18] G. M. Lieberman, The Perron process applied to oblique derivative problems, Adv. Math. 55 (1985), 161-172. 
[19] G. M. Lieberman, Intermediate Schauder estimates for oblique derivative problems, Arch. Ration. Mech. Anal. 93 (1986), no. 2, 129-134.

[20] G. M. Lieberman, Mixed boundary value problems for elliptic and parabolic differential equations of second order, J. Math. Anal. Appl. 113 (1986), 422-440.

[21] G. M. Lieberman, Optimal Hölder regularity for mixed boundary value problems, J. Math. Anal. Appl. 143 (1989), 572-586.

[22] T. Marquardt, The inverse mean curvature flow for hypersurfaces with boundary, Ph.D. thesis, FU Berlin, 2012.

[23] T. Marquardt, Inverse mean curvature flow for star-shaped hypersurfaces evolving in a cone, J. Geom. Anal. 23 (2013), 1303-1313.

[24] U. Massari, Esistenza e regolarita delle ipersuperfici di curvatura media assegnata in $\mathbb{R}^{n}$, Arch. Ration. Mech. Anal. 55 (1974), 357-382.

[25] R. Moser, The inverse mean curvature flow and $p$-harmonic functions, J. Eur. Math. Soc. (JEMS) 9 (2007), 77-83.

[26] R. Schoen and S.-T. Yau, On the proof of the positive mass conjecture in general relativity, Comm. Math. Phys. 65 (1979), 45-76.

[27] F. Schulze, Nichtlineare Evolution von Hyperflächen entlang ihrer mittleren Krümmung, Ph.D. thesis, Universität Tübingen, 2002.

[28] F. Schulze, Nonlinear evolution by mean curvature and isoperimetric inequalities, J. Differential Geom. 79 (2008), 197-241.

[29] L. M. Simon, Lectures on geometric measure theory, Proc. Centre Math. Appl. Austral. Nat. Univ. 3, Australian National University, Canberra 1983.

[30] A. Stahl, Convergence of solutions to the mean curvature flow with a Neumann boundary condition, Calc. Var. Partial Differential Equations 4 (1996), no. 5, 421-441.

[31] A. Stahl, Regularity estimates for solutions to the mean curvature flow with a Neumann boundary condition, Calc. Var. Partial Differential Equations 4 (1996), no. 4, 385-407.

[32] I. Tamanini, Boundaries of Caccioppoli sets with Hölder-continuous normal vector, J. reine angew. Math. 334 (1982), 27-39.

[33] J. Urbas, On the expansion of starshaped hypersurfaces by symmetric functions of their principal curvatures, Math. Z. 205 (1990), 355-372.

[34] A. Volkmann, Free boundary problems governed by mean curvature, Ph.D. thesis, FU Berlin, 2014.

[35] V. Vulcanov, Mean curvature flow of graphs with free boundaries, Ph.D. thesis, FU Berlin, 2011.

Thomas Marquardt, Department of Mathematics, ETH Zurich, Rämistrasse 101, 8092, Zurich, Switzerland e-mail: thomas.marquardt@math.ethz.ch

Eingegangen 30. April 2014, in revidierter Fassung 31. August 2014 